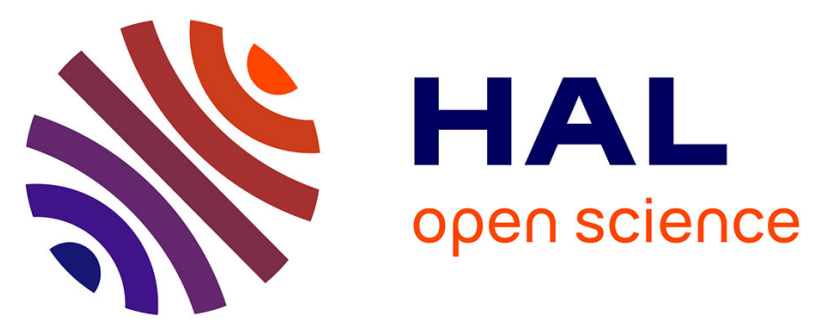

\title{
The importance of Thermo-Hydro-Mechanical couplings and microstructure to strain localization in 3D continua with application to seismic faults. Part II: Numerical implementation and post-bifurcation analysis
} Hadrien Rattez, Ioannis Stefanou, Jean Sulem, Manolis Veveakis, Thomas Poulet

\section{To cite this version:}

Hadrien Rattez, Ioannis Stefanou, Jean Sulem, Manolis Veveakis, Thomas Poulet. The importance of Thermo-Hydro-Mechanical couplings and microstructure to strain localization in 3D continua with application to seismic faults. Part II: Numerical implementation and post-bifurcation analysis. Journal of the Mechanics and Physics of Solids, 2018, 10.1016/j.jmps.2018.03.003 . hal-01728837

\section{HAL Id: hal-01728837 \\ https://hal-enpc.archives-ouvertes.fr/hal-01728837}

Submitted on 12 Mar 2018

HAL is a multi-disciplinary open access archive for the deposit and dissemination of scientific research documents, whether they are published or not. The documents may come from teaching and research institutions in France or abroad, or from public or private research centers.
L'archive ouverte pluridisciplinaire HAL, est destinée au dépôt et à la diffusion de documents scientifiques de niveau recherche, publiés ou non, émanant des établissements d'enseignement et de recherche français ou étrangers, des laboratoires publics ou privés. 


\title{
The importance of Thermo-Hydro-Mechanical couplings and microstructure to strain localization in 3D continua with application to seismic faults. Part II: Numerical implementation and post-bifurcation analysis
}

\author{
Hadrien Rattez ${ }^{\mathrm{a}}$, Ioannis Stefanou ${ }^{\mathrm{a}, *}$, Jean Sulem $^{\mathrm{a}}$, Manolis Veveakis ${ }^{\mathrm{b}, \mathrm{c}}$, \\ Thomas Poulet ${ }^{\mathrm{b}, \mathrm{c}}$ \\ ${ }^{a}$ Laboratoire Navier, UMR 8205, École des Ponts ParisTech, IFSTTAR, CNRS, \\ Champs-sur-Marne, France \\ ${ }^{b}$ School of Petroleum Engineering, University of New South Wales, Sydney, Australia \\ ${ }^{c}$ CSIRO Mineral Resources Flagship, North Ryde, NSW 2113, Australia
}

\begin{abstract}
In this paper, we study the phenomenon of localization of deformation in fault gouges during seismic slip. This process is of key importance to understand frictional heating and energy budget during an earthquake. A infinite layer of fault gouge is modeled as a Cosserat continuum taking into account ThermoHydro-Mechanical (THM) couplings. The theoretical aspects of the problem are presented in the companion paper (Rattez et al., 2017a), together with a linear stability analysis to determine the conditions of localization and estimate the shear band thickness. In this Part II of the study, we investigate the postbifurcation evolution of the system by integrating the full system of non linear equations using Finite Elements.

The problem is formulated in the framework of Cosserat theory. It enables to introduce information about the microstructure of the material in the constitutive equations and to regularize the mathematical problem in the postlocalization regime. We emphasize the influence of the microstructure and of the softening law on the material response and the strain localization process. The
\end{abstract}

\footnotetext{
${ }^{*}$ Corresponding author

Email address: ioannis.stefanou@enpc.fr (Ioannis Stefanou)
} 
weakening effect of pore fluid thermal pressurization induced by shear heating is examined and quantified. It enhances the weakening process and contributes to the narrowing of shear band thickness. Comparisons with the linear analysis exhibit that when the perturbed field of shear deformation dominates, the estimation of the shear band thickness obtained from linear stability analysis differs from the one obtained from the finite element computations, demonstrating the importance of post-localization numerical simulations.

Keywords: Cosserat continuum, Finite Element model, Thermo-Hydro-Mechanical couplings, Strain localization, Fault mechanics

\section{Introduction}

Field observations of fault zones show that, during seismic slip, shear deformation is localized in a very thin zone of finite thickness, which is called Principal Slip Zone (PSZ) (see Sibson (2003), Rempe et al. (2017), Rattez et al. 5 (2017a) for a detailed discussion). This phenomenon is favored by weakening of the gouge material and it is enhanced by multi-physical couplings (Sulem and Stefanou, 2016). Determining the size of the localized zone is a key issue for understanding the role of the different mechanisms and their impact on earthquakes (Kanamori and Brodsky, 2004).

The thickness of the localization zone was investigated using Linear Stability Analysis (LSA) in the companion paper (Rattez et al., 2017a). This analytical approach allowed to explore qualitatively the influence of various (bifurcation) parameters of the gouge layer, such as the grain size, the hardening modulus, the friction and dilatancy angles, the thermal pressurization coefficient, the normal 15 stress, the porosity and the permeability.

However, the aforementioned investigation is based on Linear Stability Analysis that has two drawbacks (see also Chambon et al. (2004)). The first one is the linearization of the system of equations, which regarding mechanics does not distinguish between loading and unloading conditions. The second one is that 
the linearization is performed on a reference state that does not evolve with deformation. These are strong assumptions for studying the post-bifurcation behavior of a non-linear system. This is why, in the present paper, the full system of non-linear equations (see Part 1) is integrated numerically, using a novel three-dimensional (3D) Finite Element (FE) code, which accounts for a 25 general, 3D Cosserat continuum formulation with Thermo-Hydro-Mechanical (THM) couplings. An elasto-plastic constitutive model that accounts for the progressive softening of the material and the grain size is implemented in the numerical code.

This numerical tool allows to point out the limitations of the LSA presented in the companion paper and offers a more accurate description of the evolution of the thickness of the localization zone. Moreover, it enables to investigate the full stress-strain response of a fault gouge, and explore its dependency on various parameters such the grain size and the applied shear rate. The precise evaluation of the stress-strain response of the fault zone under THM couplings

35 is of key importance for studying earthquake nucleation as the softening rate controls the transition from aseismic to seismic slip (Tse and Rice, 1986, Scholz, 2002).

It is worth emphasizing that computing the evolution of strain localization is a challenging task due to the difficulties that arise when dealing with soft40 ening behavior. It entails a loss of ellipticity of the governing equations in the classical continuum theory framework (de Borst et al., 1993, Vardoulakis and Sulem, 1995). The boundary value problem becomes mathematically ill-posed (Vardoulakis, 1986) and the results of classical finite element computations exhibit a mesh dependency (as deformations localize in a band of thickness equal 45 to the finite element size). Moreover, mesh refinement leads towards zero energy dissipation, which is nonphysical. However, the Cosserat FE formulation followed herein does not suffer from the above issues as it possesses a material parameter with dimension of length which regularizes the numerical problem (see also de Borst (1991), Godio et al. (2016), Stefanou et al. (2017)).

Besides Cosserat, different methods have been developed to address the 
problem of mesh dependency: viscoplastcity (but only under dynamic loading conditions) (Needleman, 1988), non-local continua (Pijaudier and Bazant, 1987), gradient plasticity (Vardoulakis and Aifantis, 1991) and continua with microstructure (Papanastasiou and Vardoulakis, 1992, Collin et al., 2006), among 55 others. As these methods are not based on the same assumptions, the choice of one or another depends on the physical mechanisms that the modeler wants to describe (Papanastasiou and Zervos, 2016) (see de Borst et al. (1993) for a detailed comparison). For instance, if non-negligible rate dependency is experimentally observed for a given material, visco-plasticity is the natural framework.

${ }_{60}$ For granular materials such as fault gouge, Cosserat continuum (a special case of continua with microstructure (Germain, 1973) appears to be the most appropriate framework (Papanastasiou and Zervos, 2016) (see also the companion paper (Rattez et al., 2017a)).

Several studies have focused on the validation of an elasto-plastic Cosserat continuum against experimental results comparing the size of the shear band for biaxial tests (Mühlhaus and Vardoulakis, 1987, Alsaleh, 2004) or other geometries (Tejchman, 2008). Some authors have also developed strategies to calibrate the parameters of the model using a series of cyclic triaxial and compression biaxial tests (Ehlers and Scholz, 2007) or a combination of micro-CT images and 70 macroscopic stress-strain response for a single triaxial test (Wang et al., 2016).

This paper is organized as follows. Section 2 describes the finite element formulation of a three dimensional (3D) Cosserat continuum with Thermo-HydroMechanical couplings as well as the the numerical implementation of the constitutive model presented in the companion paper (Rattez et al., 2017a). The formulation is kept general in order to allow various applications in geomechanics. In Section 3, the particular problem of an infinite sheared layer without THM couplings is presented. The limitations of the classical Cauchy continuum are discussed and the advantages of the Cosserat continuum are emphasized for modeling the behavior of a fault gouge. The influence of the main constitutive parameters and of the size of the microstructure on the stress-strain diagram and on strain localization evolution is examined. In Section 4, the THM cou- 
plings are introduced and their impact on the overall behavior of a fault gouge is investigated. The role of thermal pressurization is highlighted. Finally, in Section 5 , the numerically obtained shear band thickness is compared to the 85 results of the LSA presented in the companion paper (Rattez et al., 2017a).

\section{Governing Equations and numerical implementation}

The governing equations of a Cosserat continuum under THM couplings are derived from the linear and angular momentum balance equations, the mass and the energy balance equations (for more details, see Sections 3 and 4 in the companion paper). They are then completed with the necessary constitutive equations, i.e. a three dimensional elasto-plastic constitutive law considering a Drucker-Prager yield surface extended for Cosserat continua with a single plastic multiplier (Godio et al., 2016).

\subsection{Formulation of a Cosserat continuum model and balance laws}

Let us consider a fluid saturated Cosserat medium that occupies a domain with volume $\Omega$ and is delimited by a boundary $\partial \Omega$. Each material point possesses six degrees of freedom, i.e. three translations $u_{i}$ and three rotations $\omega_{i}^{c}$. For the strain rate tensor, $\dot{\gamma}_{i j}$, and the curvature rate tensor, $\dot{\kappa}_{i j}$, a decomposition into an elastic, a plastic and a thermal part is assumed:

$$
\dot{\gamma}_{i j}=\dot{\gamma}_{i j}^{e}+\dot{\gamma}_{i j}^{p}+\dot{\gamma}_{i j}^{t h} \quad \text { and } \quad \dot{\kappa}_{i j}=\dot{\kappa}_{i j}^{e}+\dot{\kappa}_{i j}^{p}
$$

100

Thermal strain rates are written as: $\dot{\gamma}_{i j}^{t h}=\alpha \dot{T} \delta_{i j}$ where $\alpha$ is the coefficient of thermal expansion and $T$ the temperature. The stress tensor $\tau_{i j}$ is in general non-symmetric and is split into a symmetric $\sigma_{i j}$ and a antisymmetric part $\tau_{[i j]}$. similarly, the deformation tensor $\gamma_{i j}$ is decomposed into a symmetric $\varepsilon_{i j}$ and an antisymmetric $\gamma_{[i j]}$ part. A couple-stress tensor $\mu_{i j}$ is introduced, which is dual in energy with the curvature tensor $\kappa_{i j}$. 
The momentum balance equations for each point inside $\Omega$ can be written as follows:

$$
\begin{array}{r}
\tau_{i j, j}=0 \\
\mu_{i j, j}-e_{i j k} \tau_{j k}=0
\end{array}
$$

where $e_{i j k}$ is the Levi-Civita symbol. Inertia terms, volumetric forces and moments are neglected herein.

The boundary is partitioned into two parts, depending on the type of boundary conditions applied, $\partial \Omega=\partial \Omega_{U}+\partial \Omega_{\Sigma}$. Neumann boundary conditions are applied on $\partial \Omega_{\Sigma}$ :

$$
\begin{gathered}
\tau_{i j} n_{j}=t_{i}^{d} \\
\mu_{i j} n_{j}=m_{i}^{d}
\end{gathered}
$$

where $t_{i}^{d}$ and $m_{i}^{d}$ are the prescribed traction and moment vectors on $\partial \Omega_{\Sigma}$ respectively. The field $n_{j}$ denotes the unit normal vector at any point of the boundary of the domain $\partial \Omega$. Dirichlet boundary conditions can be prescribed on the boundary $\partial \Omega_{U}$ :

$$
\begin{gathered}
u_{i}=u_{i}^{d} \\
\omega_{i}^{c}=\omega_{i}^{c d}
\end{gathered}
$$

where $u_{i}^{d}$ and $\omega_{i}^{c d}$ are the prescribed displacement and rotation vectors on $\partial \Omega_{U}$ respectively.

By assuming that all the plastic work is converted into heat and that the heat flux is expressed through Fourier's law, we obtain the following diffusion equation for the temperature $T$ in $\Omega$ (Stefanou et al., 2017):

$$
\rho C\left(\frac{\partial T}{\partial t}-c_{t h} T_{, i i}\right)=\sigma_{i j} \dot{\varepsilon}_{i j}^{p}+\tau_{[i j]} \dot{\gamma}_{[i j]}^{p}+\mu_{i j} \dot{\kappa}_{i j}^{p}
$$

where $c_{t h}$ is the thermal diffusivity and $\rho \mathrm{C}$ is the specific heat per unit volume of the material. 
The diffusion equation for the pore pressure, $p$, is obtained from the fluid mass balance equation in $\Omega$ :

$$
\frac{\partial p}{\partial t}=c_{h y} p_{, i i}+\Lambda \frac{\partial T}{\partial t}-\frac{1}{\beta^{*}} \frac{\partial \varepsilon_{v}}{\partial t}
$$

where $c_{h y}$ is the hydraulic diffusivity, $\Lambda=\frac{\lambda^{*}}{\beta^{*}}$ is the thermal pressurization coefficient, $\lambda^{*}=n \lambda^{f}+(1-n) \lambda^{s}$, with $\lambda^{f}$ the fluid thermal expansion coefficient, $\lambda^{s}$ the solid thermal expansion coefficient, $\beta^{*}=n \beta^{f}+(1-n) \beta^{s}$, with $\beta^{f}$ the fluid compressibility and $\beta^{s}$ the compressibility of the solid phase (Rattez et al., 2017a).

In the right hand side of Eq. 2.7, the source term for thermal pressurization of the pore fluid is controled by the coefficient $\Lambda$. Thermal pressurization of the pore fluid is a weakening mechanism for the shear stress that can enhance strain localization.

Assuming a Drucker-Prager yield surface and plastic potential, the elastoplastic incremental generalized stress-strain relationships are written as follows:

$$
\begin{aligned}
& {\dot{\tau^{\prime}}}_{i j}=C_{i j k l}^{e p} \dot{\gamma}_{k l}+D_{i j k l}^{e p} \dot{\kappa}_{k l}+E_{i j k l}^{e p} \dot{T} \delta_{k l} \\
& \dot{\mu}_{i j}=M_{i j k l}^{e p} \dot{\kappa}_{k l}+L_{i j k l}^{e p} \dot{\gamma}_{k l}+N_{i j k l}^{e p} \dot{T} \delta_{k l}
\end{aligned}
$$

where $C_{i j k l}^{e p}, D_{i j k l}^{e p}, E_{i j k l}^{e p}, M_{i j k l}^{e p}, N_{i j k l}^{e p}$ and $L_{i j k l}^{e p}$ are fourth-order constitutive tensors derived in the companion paper (Rattez et al., 2017a). $\tau_{i j}^{\prime}$ is the Terzaghi effective stress tensor linked to the total stress tensor by $\tau_{i j}^{\prime}=\tau_{i j}+p \delta_{i j}$ (compression negative). For the sake of simplicity, incompressible solid skeleton is assumed here. However, a formulation for compressible solid skeleton within the frame of Biot theory of poromechanics (Rice, 1975) is straightforward to extend to Cosserat continua but exceeds the scope of the present work.

\subsection{Finite element implementation and validation}

The full system of equations 2.2-2.1 is integrated numerically. A displacementrotation-temperature-pore pressure incremental finite element formulation is used. The integration in time is implicit using the backward Euler method, 
which is implemented in Redback (Poulet et al., 2016), a module of the Finite Element framework MOOSE (Gaston et al., 2009).

The weak form of the linear and angular momentum balance equations Eqs.2.2-2.3 is written Godio et al. (2015) using Green's identity:

$$
\begin{aligned}
& -\int_{\Omega} \tau_{i j} \psi_{i, j} d \Omega+\int_{\partial \Omega_{\Sigma}} \tau_{i j} n_{j} \psi_{i} d S=0 \\
& -\int_{\Omega} \mu_{i j} \psi_{i, j} d \Omega+\int_{\partial \Omega_{\Sigma}} \mu_{i j} n_{j} \psi_{i} d S-\int_{\Omega} \varepsilon_{i j k} \tau_{j k} \psi_{i} d \Omega=0
\end{aligned}
$$

The energy and fluid mass balance equations are also written in their weak forms:

$$
\begin{array}{r}
\int_{\Omega} \dot{p} \psi d \Omega+c_{h y}\left(\int_{\Omega} p_{, i} \psi, i d \Omega-\int_{\partial \Omega} p_{, i} n_{i} \psi d S\right)-\Lambda \int_{\Omega} \dot{T} \psi d \Omega+\frac{1}{\beta^{*}} \int_{\Omega} \dot{\varepsilon_{v}} \psi d \Omega=0 \\
\int_{\Omega} \dot{T} \psi d \Omega+c_{t h}\left(\int_{\Omega} T_{, i} \psi_{, i} d \Omega-\int_{\partial \Omega} T_{, i} n_{i} \psi d S\right)-\frac{1}{\rho C} \int_{\Omega}\left(\tau_{i j} \dot{\gamma}_{i j}^{p}+\mu_{i j} \dot{\kappa}_{i j}^{p}\right) \psi_{, i} d \Omega=0
\end{array}
$$

where $\psi$ and $\psi_{i}$ are the test functions. $c_{h y}, c_{t h}, \rho C, \beta^{*}$ and $\Lambda$ are considered constant herein.

Linear Lagrange test functions are chosen for all the fields and full integration is performed. An alternative choice would be to use quadratic functions with reduced integration to improve the rate of mesh convergence (Godio et al., 2015), but they are not used here for simplicity. Nevertheless, mesh convergence is verified in all the analyses presented in this paper (see Section 3.1).

In order to validate the Finite Element implementation and all the couplings, a systematic procedure was followed. In particular, the implementation of each term for Eqs. 2.9-2.12 is verified through appropriate unit tests and comparisons 


\section{Simple shear of a layer without THM couplings}

In this section, we present the problem of localization of deformation in a sheared layer by considering a geometry and parameters that are consistent with a gouge in a fault core. Only the mechanical equations for an elastoplastic Cosserat continuum are considered (without couplings). The results are compared to the ones already published (de Borst, 1991, Tejchman, 2008, Godio et al., 2016). The methodology for the evaluation of the shear band thickness is presented. Emphasis is given to describing the influence of the internal length and different softening laws on the localization and the response of the material. These results and investigations are then used as a reference to study the influence of the Thermo-Hydro Mechanical couplings (Section 4).

\subsection{Problem statement and mesh convergence}

The fault core is modeled as an infinite layer of height $h$ subjected to shear under constant velocity $V$ and normal stress $\tau_{n}$, as shown in Fig. 1 . The values of the different parameters are chosen to represent a fault gouge at a seismogenic depth of $7 \mathrm{~km}$, which is a typical centroidal depth for crustal faults. Furthermore, as this set of parameters was used in previous studies, we can compare our results to foregoing works on the subject and complement them (Rice, 2006, Sulem et al., 2011, Platt et al., 2014). The rotations are imposed at the upper and lower boundaries $\left(\omega_{3}^{c}=0\right)$ as it entails the development of a single band in the middle of the layer and facilitates the comparisons of stress-strain diagrams as explained in Appendix B.

The Drucker-Prager yield criterion is generalized for Cosserat continua, as in Rattez et al. (2017a) with a friction coefficient which evolves with plastic shear deformation:

$$
F=\tau+\mu \cdot \sigma^{\prime}-c
$$

where $\sigma^{\prime}=\frac{\tau_{k k}^{\prime}}{3}, c$ is the cohesion and $\tau$ is the generalized second invariant of 


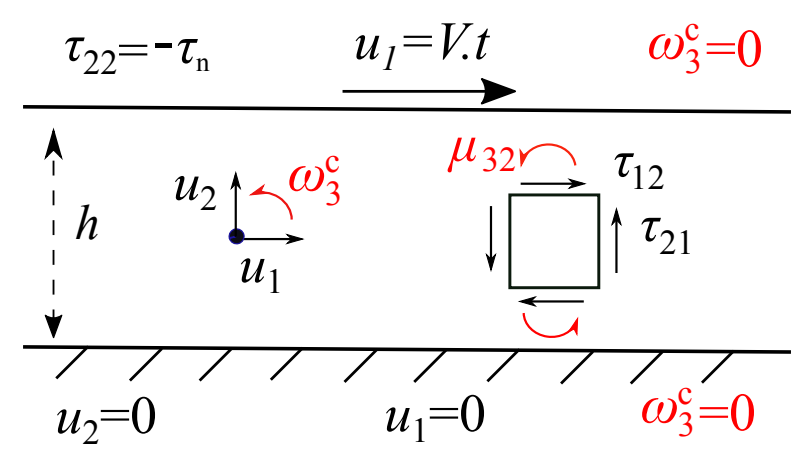

Figure 1: Infinite layer of a fault material under shear. Notations and boundary conditions.

the deviatoric stress tensor, defined by:

$$
\tau=\sqrt{h_{1} s_{i j} s_{i j}+h_{2} s_{i j} s_{j i}+\frac{1}{R^{2}}\left(h_{3} m_{i j} m_{i j}+h_{4} m_{i j} m_{j i}\right)}
$$

where $s_{i j}, m_{i j}$ are the deviatoric parts of the stress and couple-stress tensors respectively. The hardening coefficient $h_{s}$ defines the evolution of the friction coefficient with the accumulated plastic shear strain:

$$
h_{s}=\frac{d \mu}{d \gamma^{p}}
$$

and is linked to the hardening modulus $H_{s}$ by the equation $H_{s}=h_{s} \sigma^{\prime}$.

The coefficients in the stress invariant are $h_{1}=h_{3}=\frac{2}{3}$ and $h_{2}=h_{4}=$ $\frac{-1}{6}$ and $R$ is the internal length of the Cosserat continuum as in (Sulem and Vardoulakis, 1990). The generalized plastic strain rate invariant is defined as:

$$
\dot{\gamma}_{p}=\sqrt{g_{1} \dot{e}_{i j}^{p} \dot{e}_{i j}^{p}+g_{2} \dot{e}_{i j}^{p} \dot{e}_{j i}^{p}+R^{2}\left(g_{3} \dot{k}_{i j}^{p} \dot{k}_{i j}^{p}+g_{4} \dot{k}_{i j}^{p} \dot{k}_{j i}^{p}\right)}
$$

where $e_{i j}$ and $k_{i j}$ are the deviatoric parts of the strain and curvature tensors respectively and $g_{1}=g_{3}=\frac{8}{5}, g_{2}=g_{4}=\frac{2}{5}$. In (Mühlhaus, 1986, Mühlhaus and Vardoulakis, 1987, Sulem and Vardoulakis, 1990), these coefficients were calculated based on micromechanical considerations. An example of calculation for one set of invarants is presented in Appendix B of the companion paper (Rattez et al., 2017a). The influence of the invariants' expression on the stress- 
strain graph is shown in Fig. 5 and the values of the different sets of coefficients are recalled in Table 1.

\begin{tabular}{|c|c|c|}
\hline & 2D model & 3D model \\
\hline Static model & $\left\{h_{i}\right\}=\{3 / 4,-1 / 4,1,0\}$ & $\left\{h_{i}\right\}=\{2 / 3,-1 / 6,2 / 3,-1 / 6\}$ \\
& $\left\{g_{i}\right\}=\{3 / 2,1 / 2,1,0\}$ & $\left\{g_{i}\right\}=\{8 / 5,2 / 5,8 / 5,2 / 5\}$ \\
\hline Kinematic model & $\left\{h_{i}\right\}=\{3 / 8,1 / 8,1 / 4,0\}$ & $\left\{h_{i}\right\}=\{2 / 5,1 / 10,2 / 5,1 / 10\}$ \\
& $\left\{g_{i}\right\}=\{3,-1,4,0\}$ & $\left\{g_{i}\right\}=\{8 / 3,-2 / 3,8 / 3,-2 / 3\}$ \\
\hline
\end{tabular}

Table 1: Values for the coefficients of the stress and strain generalized deviatoric second invariants for a Cosserat continuum from Mühlhaus (1986), Mühlhaus and Vardoulakis (1987), Sulem and Vardoulakis (1990), Unterreiner (1994)

In this purely mechanical example, a linear softening rule is chosen to illustrate strain localization. In the numerical examples, $\mathrm{h}=1 \mathrm{~mm}$, the cohesion of the material is chosen equal to $c=100 \mathrm{kPa}$ and a linear softening rule with a hardening coefficient equal to $h_{s}=-0.5$.

The elastic parameters of the material are $K=20000 \mathrm{MPa}, G=10000$ $\mathrm{MPa}$ and $G_{c}=5000 \mathrm{MPa}$. The internal length of the Cosserat continuum is chosen to be $R=10 \mu \mathrm{m}$, which is an average grain size for highly finely granulated (ultra-cataclastic) fault core (Chester and Chester, 1998, Rice and Cocco, 2007). An initial isotropic state of stress is applied to the layer, such that $\sigma=-133.33 \mathrm{MPa}$. The values of the parameters are summarized in Table 1952.

Mesh convergence is first investigated for the considered Cosserat model. A 3D geometry is considered with periodic boundary conditions for the lateral sides of the specimen, which results in a 1D problem equivalent to the problem presented in Fig. 1. A regular mesh with hexahedric elements is chosen with a single element in directions $x_{1}$ and $x_{3}$, and a range of 40 to 240 elements in the vertical direction $x_{2}$. Given the periodic boundary conditions and the choice of the shape functions, the invariance in the $x_{1}$ and $x_{3}$ directions is guaranteed. In Fig. 2, the shear stress $\tau_{12}$ at the top of the layer is plotted versus the normalized 


\begin{tabular}{|c|c|c||c|c|c|}
\hline parameters & values & units & parameters & values & units \\
\hline$K$ & $20 \times 10^{3}$ & $\mathrm{MPa}$ & $\mu$ & 0.5 & \\
$G$ & $10 \times 10^{3}$ & $\mathrm{MPa}$ & $\beta$ & 0 & \\
$G c$ & $5 \times 10^{3}$ & $\mathrm{MPa}$ & $c$ & 100 & $\mathrm{kPa}$ \\
$R$ & 10 & $\mu \mathrm{m}$ & $h_{s}$ & -0.5 & \\
\hline
\end{tabular}

Table 2: Numerical values of the mechanical parameters of a deep rock gouge from Sulem et al. (2011), Rice (2006)

horizontal displacement at the top. As expected, the plastic regime is reached for $\tau_{12}=\mu \sigma$ and followed by a softening behavior. The results for 160 and 240 elements in the vertical direction exhibit no clear difference, indicating a mesh-convergence.

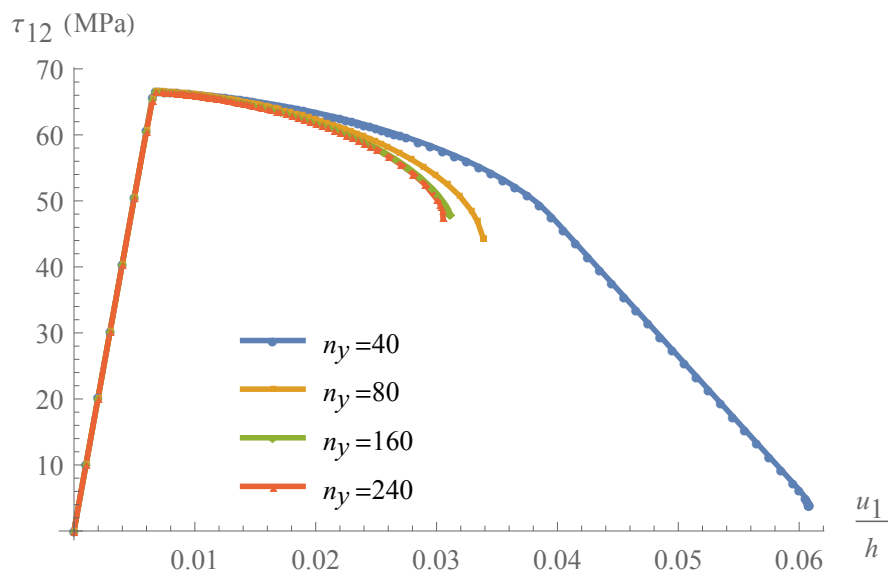

Figure 2: Stress-strain diagram obtained for an elasto-plastic infinite sheared layer modeled as a Cosserat continuum for different numbers of elements in the vertical direction

In Fig. 3, the Cosserat rotation $\omega_{3}^{c}$ is plotted on the deformed mesh with 80 elements in the vertical direction (not finer, for a clearer visualization) at the last timestep. The magnitude of the rotations is higher inside the zone of localized deformations as observed experimentally for granular materials (Hall et al., 2010). 


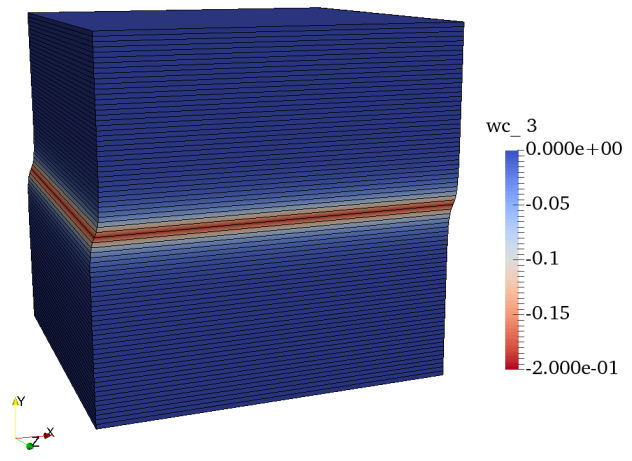

Figure 3: Cosserat rotation with 80 elements in the vertical direction for $\tau_{12}=48 \mathrm{MPa}$

In Fig. 4, the total shear strain $\gamma_{12}$ is plotted along the height of the layer for different space discretizations and a shear stress $\tau_{12}=48 \mathrm{MPa}$. This graph shows that when the mesh is fine enough, the shear band thickness is indeed meshindependent which is a key feature of the Cosserat model. The deformation profile is almost identical for 80 and 160 elements. The profile for 240 elements is not represented in this graph as it coincides with the one obtained with the mesh of 160 elements.

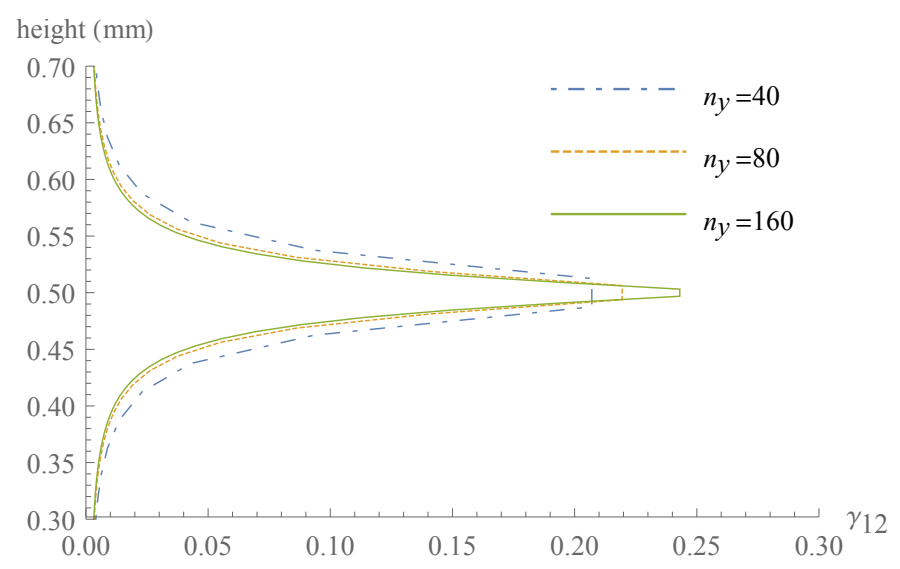

Figure 4: Mesh-independency of the shear strain profile for $\tau_{12}=48 \mathrm{MPa}$ 


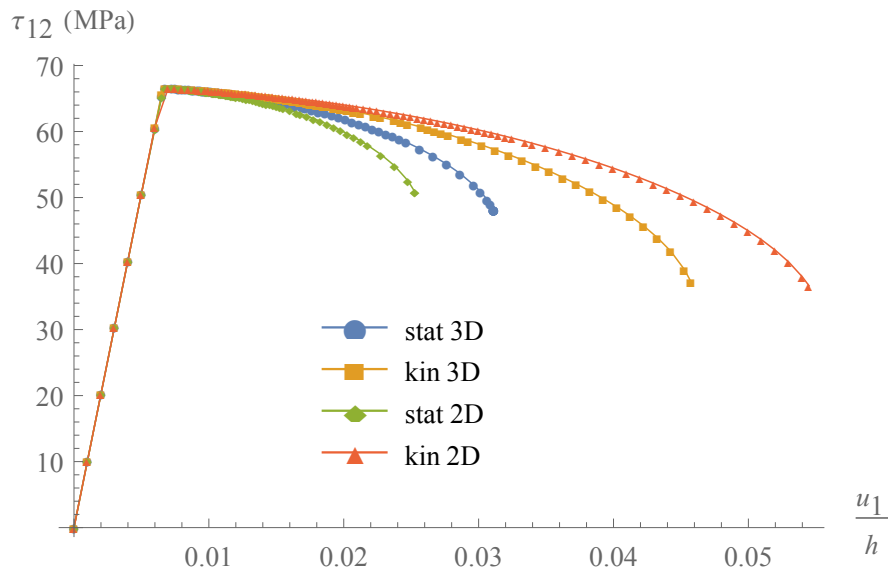

Figure 5: Stress-strain graph for converged meshes computed with different values of the stress and strain generalized invariants (see Table 1)

The results obtained using a classical Cauchy continuum are retrieved in the particular case of $G_{c} \rightarrow 0$, and $R \rightarrow 0$, as explained in Iordache and William (1998). In Fig. 6, the shear stress is plotted as a function of the normalized horizontal displacement at the top.

This diagram shows the dependency of the softening branch on the mesh size. As expected, the plastic deformations localize in a single hexahedric element (see Fig. 7) and the smaller the mesh size is, the steeper the softening branch becomes. Note that the shear band is not located in the middle of the layer. As no imperfection is introduced to restrict its position, it appears "randomly" in the system due to numerical approximations.

This mesh-dependency of the load-displacement diagram has a consequence on energy dissipation. To investigate this effect, we calculate for different discretizations of the layer the plastic part of the mechanical energy, $E_{p}$, and the elastic part, $E_{e}$, both with a Cosserat and a Cauchy continuum. The elastic energy is evaluated by considering an unloading for $\tau_{12}=48 \mathrm{MPa}$. Elastic and plastic parts of the mechanical energy are shown in Fig. 8.

The energy partition is computed for different numbers of elements (Fig. 9). 


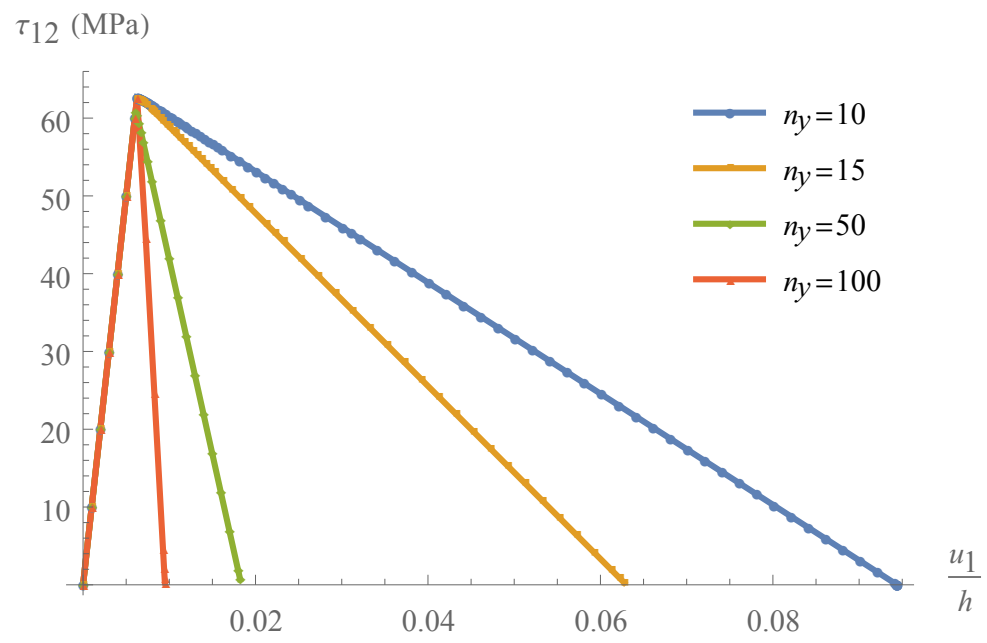

Figure 6: Stress-strain diagram obtained for an elasto-plastic infinite sheared layer modeled as a classical Cauchy continuum for different numbers of elements in the vertical direction

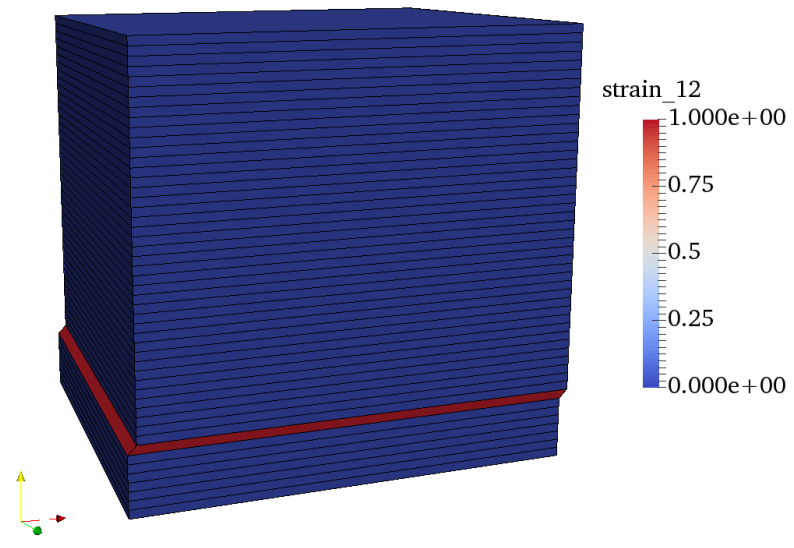

Figure 7: shear strain plotted on the deformed mesh with 50 elements $\tau_{12}=1 \mathrm{MPa}$ with a Cauchy continuum 

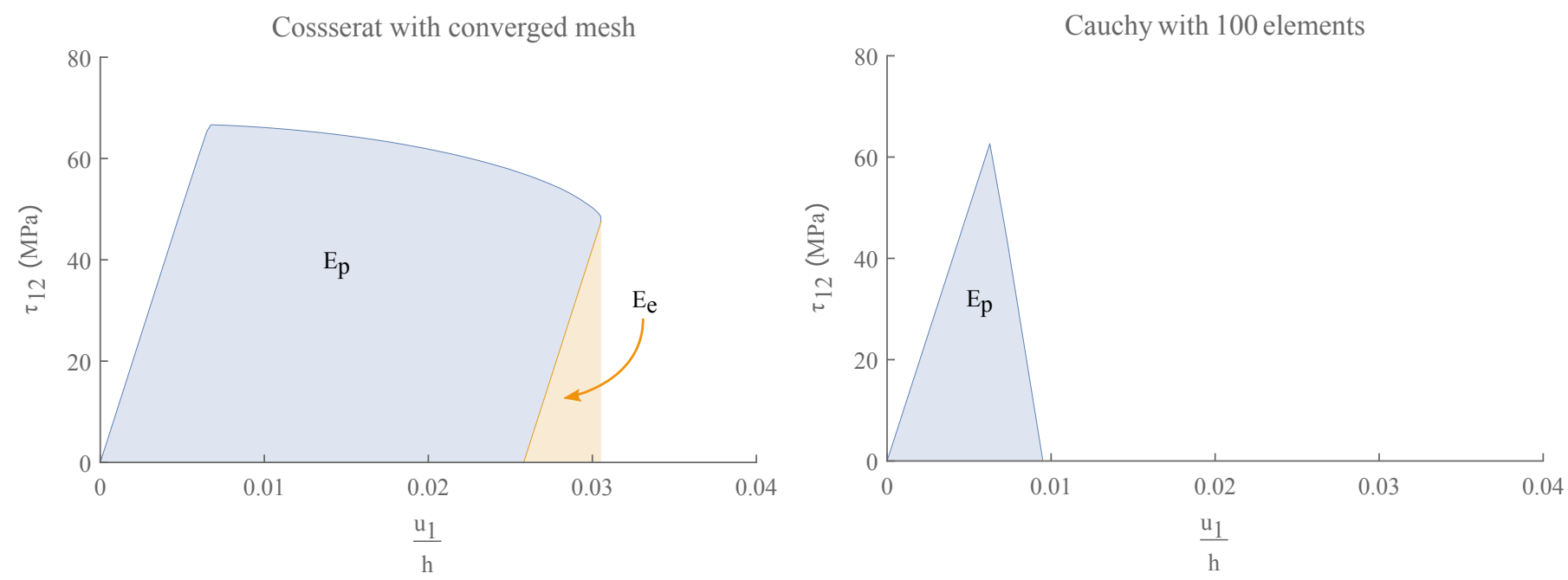

Figure 8: Elastic (Ee) and plastic (Ep) parts of the mechanical energy for a Cosserat continuum with 240 elements in the vertical direction and a Cauchy continuum with 100 elements in the vertical direction. 
For the Cosserat formulation, the plastic part of the mechanical energy tends to a constant value when the mesh size is small enough, whereas for a Cauchy continuum, the plastic energy tends to the total mechanical energy at peak. This is due to the fact that the softening branch gets steeper while increasing the number of elements. To complete the analysis of the Cauchy continuum with more elements an arc-length algorithm is necessary. It enables us to capture a "snap-back" behavior and the plastic energy will tend to zero (de Borst, 1991). physical results. It is even more problematic when Thermo-Mechanical couplings are incorporated in the model because the amount of heat produced is calculated from the plastic dissipation (Eq. 2.6).

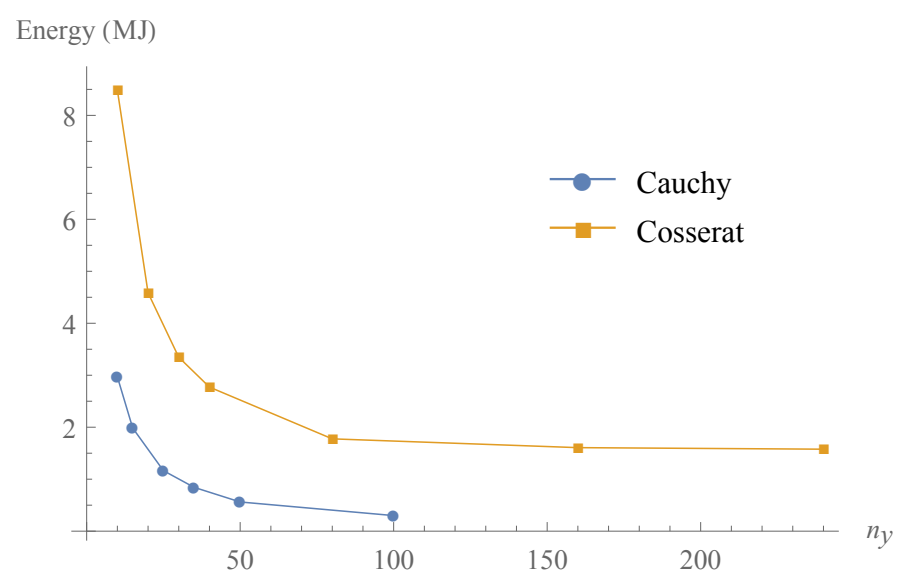

Figure 9: Computed dissipation as a function of the number of elements in the vertical direction $\left(n_{y}\right)$ for a Cosserat continuum and a Cauchy continuum

\subsection{Evaluation of the shear band thickness and sensitivity analysis}

As stated in the companion paper (Rattez et al., 2017a), the shear band thickness is a key parameter for assessing the energy dissipation when localization occurs. In order to compare the results of the linear stability analysis with the numerical simulations, we need to define a measure of this thickness.

For defining this measure we refer to the profile of the plastic strain rate 
for the evaluation of the shear band thickness, as explained below, but also for comparing numerical results with experimental ones that are obtained by Digital Image Correlation (Hall et al., 2010) $\left(\dot{\gamma}\left(x_{2}\right) \approx \dot{\gamma}^{p}\left(x_{2}\right)\right.$ inside the shear band). Furthermore, it provides a better representation of the localization process at $\omega_{i}^{c}$, whose distributions strongly depend on the stress path and history of the system.

Inside the shear band the computed plastic shear strain increment can be interpolated accurately by a cosine function, whose wavelength is defined here as the thickness of the localization zone:

$$
\dot{\gamma}^{p}\left(x_{2}\right) \approx B \cdot \chi_{\left[Y-\frac{\lambda}{2} ; Y+\frac{\lambda}{2}\right]}\left(x_{2}\right) \cdot\left[\cos \left(2 \pi \frac{\left(x_{2}-Y\right)}{\lambda}\right)+1\right]
$$

where $B$ is half of the maximum plastic strain rate, $Y$ is the position of the center, $\lambda$ is the wavelength of the cosine function and $\chi_{\left[Y-\frac{\lambda}{2} ; Y+\frac{\lambda}{2}\right]}\left(x_{2}\right)$ is a rectangular function defined by:

$$
\chi_{\left[Y-\frac{\lambda}{2} ; Y+\frac{\lambda}{2}\right]}\left(x_{2}\right)= \begin{cases}1 & \text { if } x_{2} \in\left[Y-\frac{\lambda}{2} ; Y+\frac{\lambda}{2}\right] \\ 0 & \text { otherwise }\end{cases}
$$

Notice, that this definition of the thickness of the localization zone allows a clear link with the results of the Linear Stability Analysis performed in (Rattez et al., 2017a). More details and arguments on the choice of this definition as well as comparisons with alternative ones found in the literature are given in Appendix A.

A key parameter to determine the localization of deformation and used as 275 a bifurcation parameter in linear stability analyses is the softening modulus $H_{s}$ (Rudnicki and Rice, 1975, Issen and Rudnicki, 2000). Thus, we investigate numerically its effect in the following. The load-displacement diagram depicted in Fig. 10 shows as expected that the higher (in absolute value) the softening modulus is, the steeper the stress-displacement response becomes. 


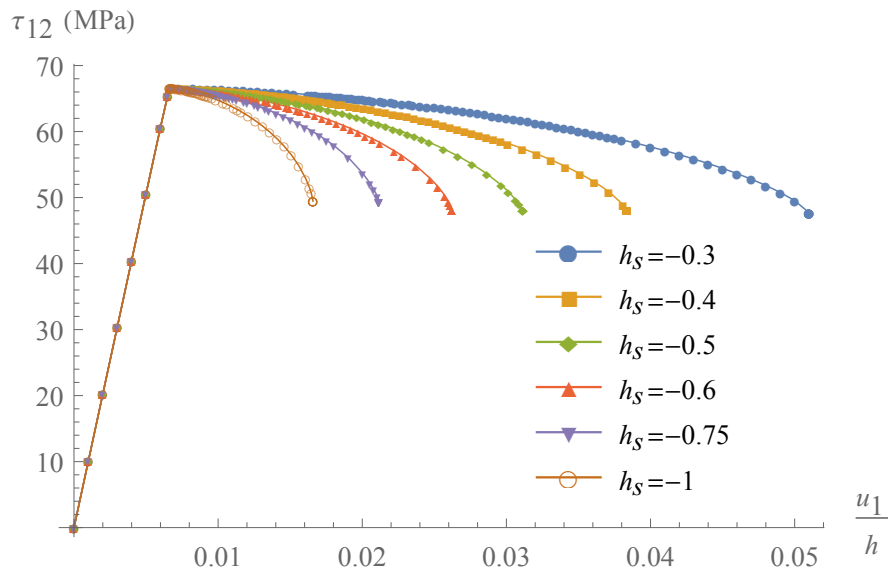

Figure 10: Effect of the hardening coefficient on the load-displacement diagram band thickness. The different shear band thickness evolutions with increasing deformation are due to the different evolutions of the shear stress (Fig. 12). 


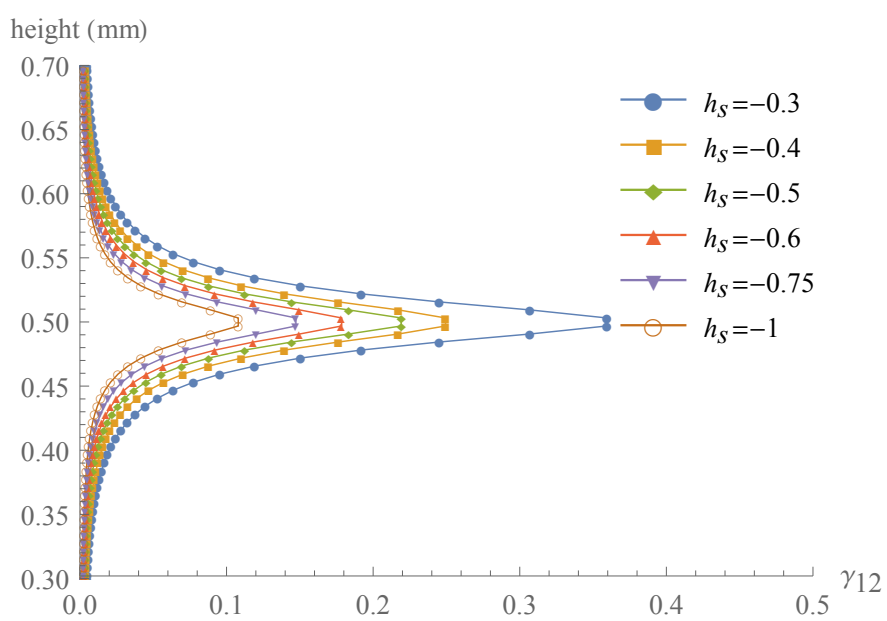

Figure 11: Effect of the hardening coefficient on the shear strain $\gamma_{12}$ profile for $\tau_{12}=48 \mathrm{MPa}$

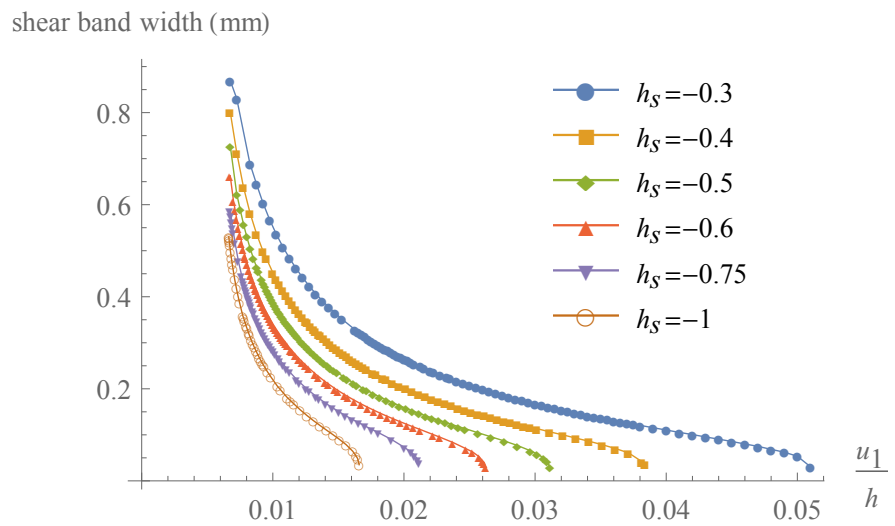

Figure 12: Effect of the hardening modulus on the shear band thickness evolution 
It is also interesting to explore how the internal length of the Cosserat model

300 shear band thickness evolution and the shear strain profile (Khoei et al., 2010, Ebrahimian et al., 2012). In Fig. 13, the load-displacement diagrams for different values of Cosserat internal length are plotted for a hardening coefficient $h_{s}=$ -0.5 . For lower values of the internal length, the softening branch of the stressdisplacement curve is steeper.

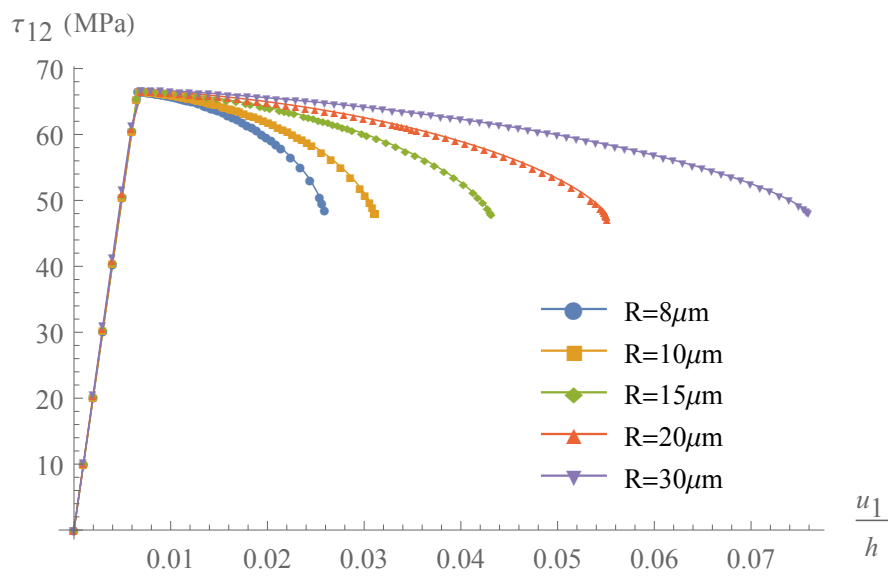

Figure 13: Effect of the internal length on the stress-strain graph

In Fig. 14, the effect of the internal length on the shear band thickness evolution is shown. For smaller internal length, shear band thickness decreases faster with increasing strain.

As shown in Fig. 15, the distribution of the shear strain $\gamma_{12}$ differs for different values of the internal length $R$. The value of the maximum is the same, but the profiles are different (unlike Fig. 12). For larger internal lengths, the shear band thickness is larger, in agreement with previous studies (Mühlhaus and Vardoulakis, 1987, Sharbati and Naghdabadi, 2006, Sulem et al., 2011). Thus, the difference of the stress-displacement curves in the post-bifurcation regime for different internal lengths is mainly due to different values of shear band thickness. 


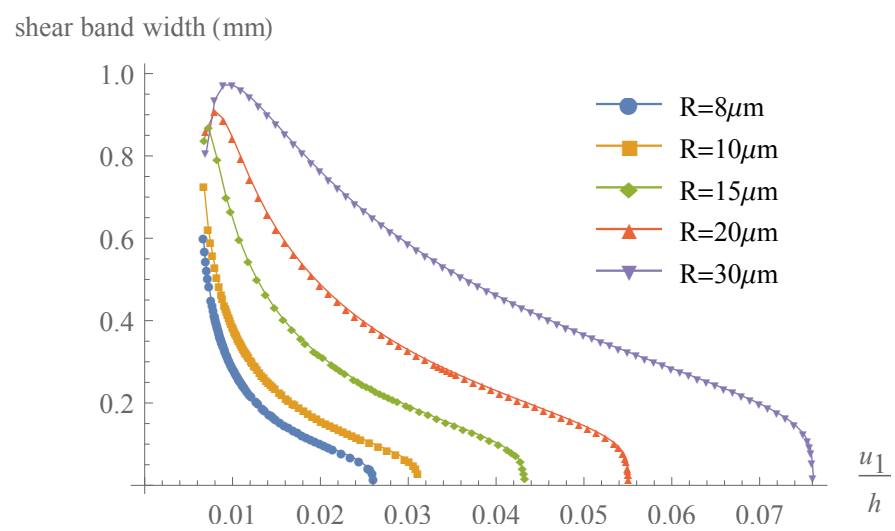

Figure 14: Effect of the internal length on the shear band thickness evolution

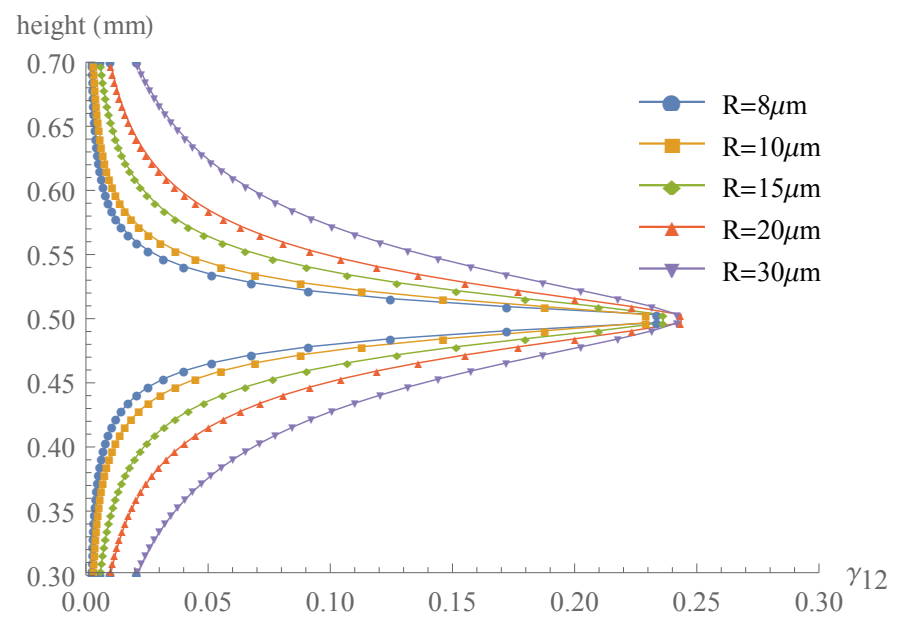

Figure 15: Effect of the internal length on the total shear strain profile for $\tau_{12}=48 \mathrm{MPa}$ 


\subsection{Exponential softening law to model granular flow}

Experimental works on granular materials have shown that a granular medium under shear evolves towards a steady state characterized by a constant friction coefficient and zero dilatancy. In the frame of classical soil mechanics, this state is called the critical state (Schofield and Wroth, 1968). Faults exhibit a similar behavior when sheared over sufficiently large distances (see Chambon et al. (2002)). This behavior has to be integrated into the constitutive description of the material in order to approximate the overall mechanical response of the system. An exponential evolution of the friction coefficient is thus assumed, which can be easily calibrated from experimental data (Mizoguchi et al., 2007, Di Toro et al., 2011, Scuderi and Collettini, 2016). In Mizoguchi et al. (2007), Di Toro et al. (2011), the authors have conducted shear experiments on simulated fault gouges at seismic slip rate and they observed an exponential decay of the friction coefficient due to various multi-physical mechanisms. For experiments performed on dry materials, these authors suggest that friction softening can be attributed to gel lubrication or nano-particles lubrication.

$$
\begin{array}{r}
\mu=\mu_{r e s}\left(1+\frac{\Delta \mu}{\mu_{r e s}} e^{-q / \gamma_{e}}\right) \\
\dot{q}=\dot{\gamma}^{p}
\end{array}
$$

where $\mu_{\text {res }}$ is the residual friction coefficient, $\Delta \mu=\mu_{i n i}-\mu_{\text {res }}$ the variation of the friction coefficient ( $\mu_{i n i}$ is the initial friction coefficient), $\gamma_{e}$ is a characteristic

320 slip weakening deformation and $q$ is the hardening variable. Alternatively to the flow theory of plasticity that is used in the present paper, a Cosserat continuum within the framework of hypoplasticity can be used to model granular materials (Huang and Bauer, 2003, Tejchman, 2008).

In Fig. 16(a), the influence of the characteristic deformation $\gamma_{e}$ is investigated. The initial friction coefficient is 0.5 and the residual one is 0.3. As expected, the bigger the factor is, the steeper the softening branch becomes. However, all the curves tend to the same asymptote around $43.73 \mathrm{MPa}$, a value higher than $\mu_{\text {res }} . \sigma=40 \mathrm{MPa}$. The reason is that the yield surface is written in 
terms of the generalized stress invariant $\tau$ (see Eq. 3.1) and not of $\tau_{12}$. This

330 into account the evolution of all the components of the stress and couple stress tensors that are especially important under multi-physical couplings. An example of the distribution of shear stresses $\tau_{12}$ and $\tau_{21}$ as well as the couple stress $\mu_{32}$ is shown in Fig. 17 for a global deformation of the layer $\frac{u_{1}}{h}$ of 1.2 for the 335

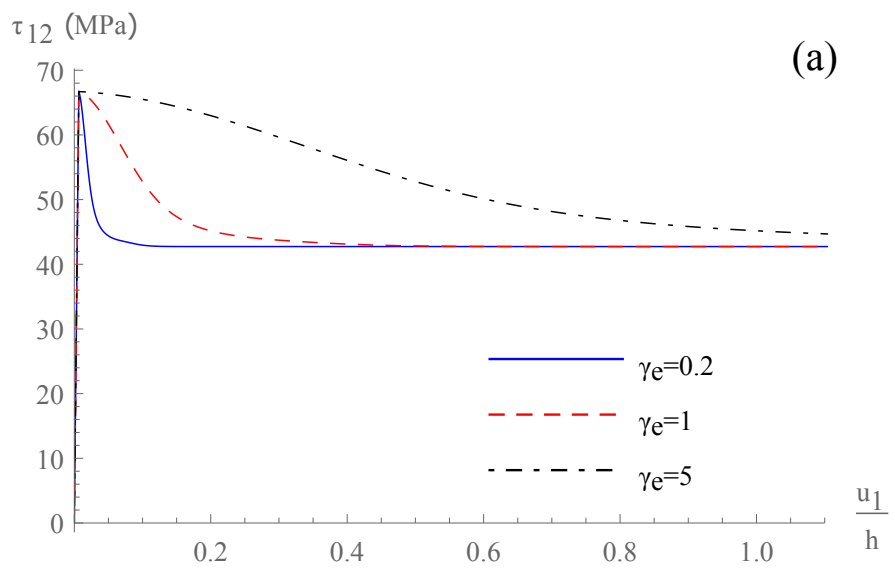

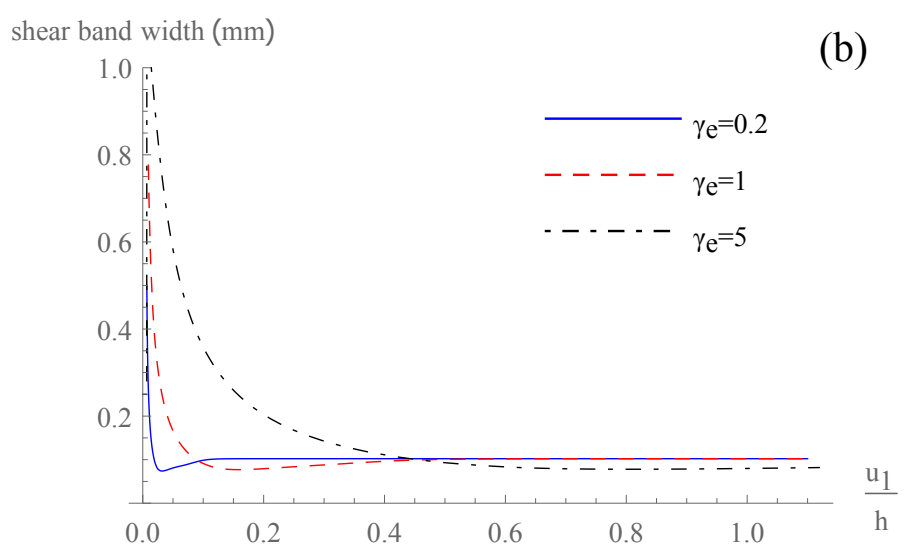

(b)

Figure 16: Effect of characteristic weakening deformation, $\gamma_{e}$, on: (a) the stress-strain diagram, (b) the shear band thickness evolution $\left(\mu_{r e s}=0.3\right)$.

In Fig. 16(b), the evolution of shear band thickness is plotted for different values of the parameter $\gamma_{e}$. We observe that, at the beginning of the localization, the shear band size decreases until a minimum that corresponds to the inflection point of the softening branch. Then, it progressively increases towards a finite value, which is independent of the $\gamma_{e}$ value. Thus, this parameter only affects the evolution of the softening behavior and the evolution of the shear band thickness but not their final values.

The stress-strain diagram for various values of the residual friction coefficient is depicted in Fig. 18(a) for a given value of the characteristic weakening deformation $\left(\gamma_{e}=1\right)$. In Fig. 18(b), the evolution of the shear band thickness with the deformation shows that the final value and also the minimum of the shear 

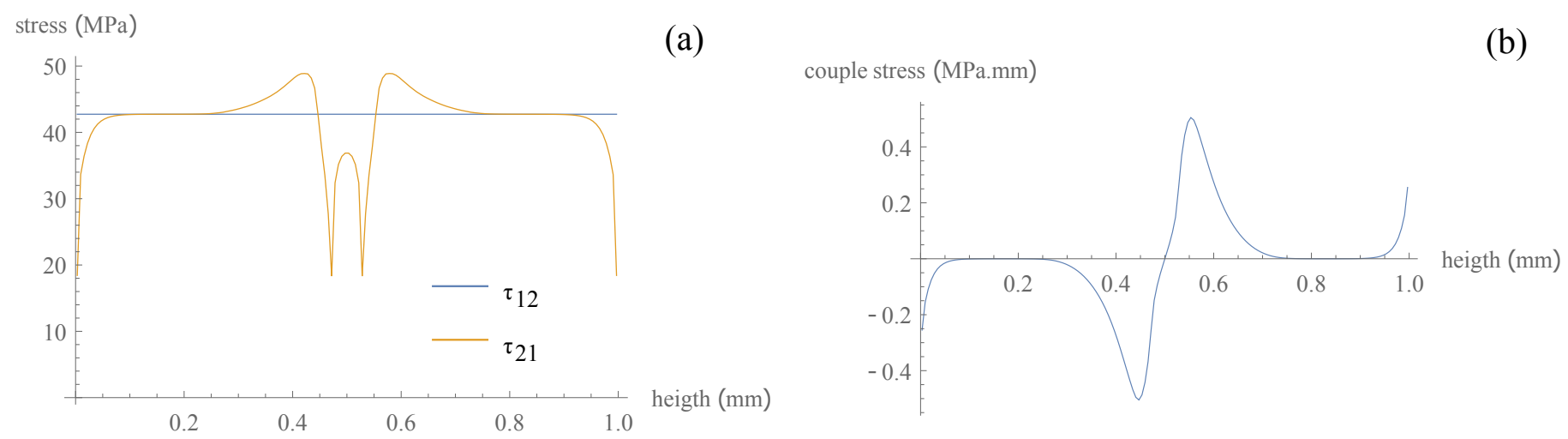

Figure 17: In the case of $\gamma_{e}=0.2$ and $\mu_{\text {res }}=0.3$.(a) Profile of the stresses $\tau_{12}$ and $\tau_{21}$ (b)

Profile of the couple stresses $\mu_{32}$
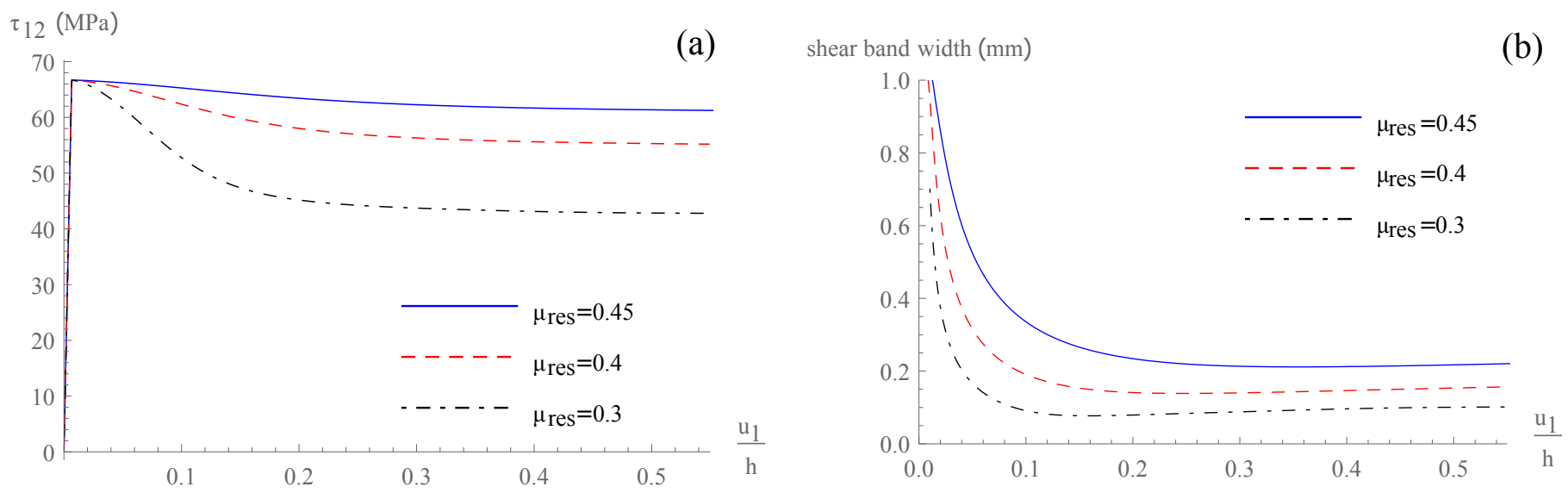

Figure 18: Effect of the residual friction, $\mu_{r e s}$, on: (a) the stress-strain diagram, (b) the shear band thickness evolution $\left(\gamma_{e}=1\right)$. 
band size are influenced by the residual friction and consequently by the total shear stress drop. A larger stress drop is associated with a thinner localized zone.
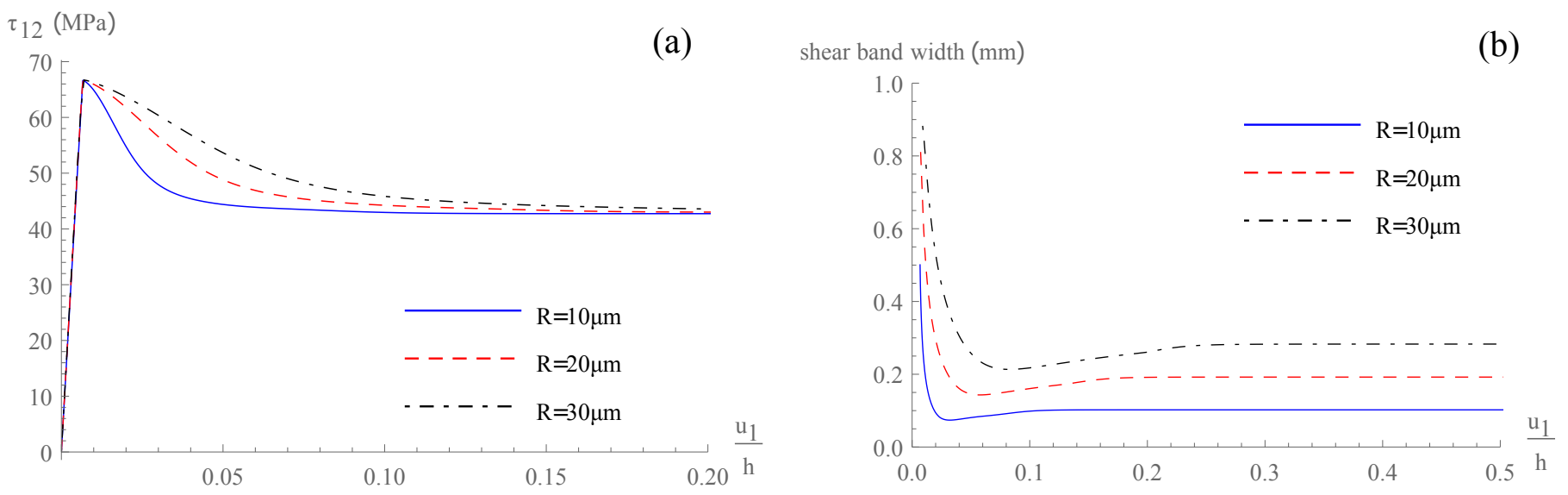

Figure 19: Effect of the internal length, R, on: (a) the stress-strain diagram, (b) the shear band thickness evolution ( $\mu_{\text {res }}=0.3$ and $\gamma_{e}=0.2$ ).

A single material length $R$ is used in the present model to represent the size of the microstructure. Previous studies on sands with a narrow grain size distribution showed a good agreement between the shear band size observed experimentally and the results obtained with a Cosserat continuum taking $R$ equal to the mean grain size (Mühlhaus and Vardoulakis, 1987, Alsaleh, 2004). However, as noted by Rice (2006), the distribution in gouge materials follows a fractal law and is much broader. Based on these observations, Sammis et al. (1987) developed a communition model to explain the generation of fault gouges. This theory is supported by experiments conducted in the laboratory (Steacy and Sammis, 1991, An and Sammis, 1994). Therefore, the use of a single material length (i.e. the mean grain size $D_{50}$ ) to describe gouge materials is an open question. However, Rice (2006) argues that the cohesion between small particles in an ultracataclasite layer would be much more important and could lead to the clustering of small particles into aggregates. This effect would raise the effective size of the microstructure to consider in the analysis (greater than 

in shear bands (Rognon et al., 2015, Tordesillas et al., 2016) imply the rotation of a set of particles and would also lead to a larger effective internal length. Therefore, a sensivity analysis on the parameter $R$ is carried out to explore its effect on the global softening response.

370 diagram (Fig. 19(a)) and the shear band thickness (Fig. 19(b)). The shear stresses tend to the same values asymptotically. However, the final values of the shear band thicknesses are different. The minimum and the residual values are plotted in Fig. 20 and we observe a linear evolution for the two estimations. A (Huang and Bauer, 2003).

In Rice (2006), the case of a principal slip zone observed in an exposure of the Punchbowl fault (Chester et al., 2005) is presented. Inside the ultacataclasite layer, a shear band with an apparent thickness of 100-300 $\mu \mathrm{m}$ seems

to have accommodated most of the slip. The mean grain size is estimated to be $D_{50}=1 \mu \mathrm{m}$. Therefore, with the set of parameters chosen here, the internal length appropriate to reproduce this pattern is one order of magnitude larger than the mean grain size (Fig. 20 ).

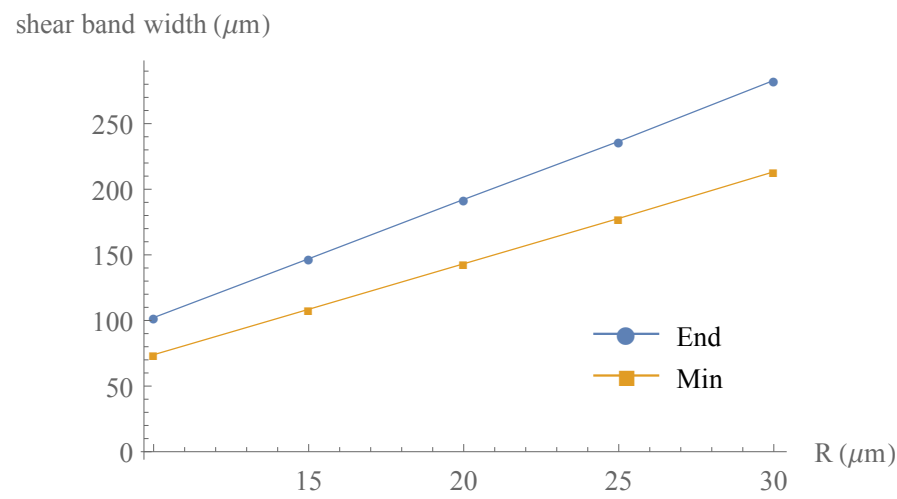

Figure 20: Evolution of the minimum and residual shear band thickness with the internal length $R$. 


\begin{tabular}{|c|c|c||c|c|c|}
\hline parameters & values & units & parameters & values & units \\
\hline$K$ & $20 \times 10^{3}$ & $\mathrm{MPa}$ & $\mu$ & 0.5 & \\
$G$ & $10 \times 10^{3}$ & $\mathrm{MPa}$ & $\beta$ & 0 & \\
$G c$ & $5 \times 10^{3}$ & $\mathrm{MPa}$ & $\lambda^{*}$ & $7.4 \times 10^{-5}$ & $/{ }^{o} \mathrm{C}$ \\
$R$ & 10 & $\mu \mathrm{m}$ & $\rho C$ & 2.8 & $\mathrm{MPa} /{ }^{o} \mathrm{C}$ \\
$\rho$ & 2500 & $\mathrm{~kg} / \mathrm{m}^{3}$ & $c_{t h}$ & 1 & $\mathrm{~mm}^{2} / \mathrm{s}$ \\
$\beta^{*}$ & $8.2 \times 10^{-5}$ & $\mathrm{MPa}^{-1}$ & $c_{h y}$ & 12 & $\mathrm{~mm}^{2} / \mathrm{s}$ \\
$n$ & 0.04 & & $\alpha_{s}$ & $2.5 \times 10^{-5}$ & $/{ }^{o} \mathrm{C}$ \\
\hline
\end{tabular}

Table 3: Numerical values for the parameters of a deep rock gouge from (Sulem et al., 2011, Rice, 2006).

\section{Effect of Thermo-Hydro-Mechanical couplings}

In the section above, the results of a dry sheared layer modeled as a Cosserat continuum have been shown in terms of stress-strain response and evolution of the shear band thickness. In this section, we consider a saturated layer and explore the effect of pore pressure and temperature changes on the strain localization process.

The numerical values for the parameters of the model refer to a saturated fault gouge at $7 \mathrm{~km}$ depth, a centroidal depth for crustal faults (Rice, 2006, Sulem et al., 2011). The mechanical conditions are the same as in Section 3 and the values for all parameters are summarized in Table 3.

An homogeneous and isotropic initial state of total stress of -200 MPa and an initial homogeneous pore pressure of $66.66 \mathrm{MPa}$ is assumed. In terms of initial effective stresses, it corresponds to $\tau_{11}^{\prime}=\tau_{22}^{\prime}=\tau_{33}^{\prime}=-133.33 \mathrm{MPa}$. A constant velocity of $1 \mathrm{~m} / \mathrm{s}$, in the range of values estimated for seismic slip (Sibson, 1973), is applied at the top of the layer.

\subsection{Influence of the various couplings}

In this section, we highlight the effects and the importance of ThermoMechanical (TM), Hydro-Mechanical (HM) and Thermo-Hydro-Mechanical (THM) 
couplings on the overall behavior of the system. The geometry and boundary conditions are kept the same as in the previous section. In Figs. 21 and 22, we present the shear stress-strain response, for $h_{s}=-0.5$ and $h_{s}=0$ respectively, of the fault gouge by activating the various couplings. The response is juxtaposed with the response of the gouge without any couplings. We observe that a clear difference is observed only when the thermal pressurization term (Eq. 2.7) is activated, i.e. for the THM model. The system under HM couplings does not show any difference compared to the purely mechanical one as no dilatancy is considered. This assumption is common for fault gouges (Sleep et al., 2000), which have already experienced significant sliding. For more details on the effect of dilatancy, we refer to the companion paper (Rattez et al., 2017a) and to (Rice, 1975, Vardoulakis, 1985, 1996, Garagash and Rudnicki, 2003, Benallal and Comi, 2003).

Regarding the TM response, it barely differs from the mechanical one. The reason is that thermal expansion is restrained by the boundary conditions and therefore it entails only a slight increase of the isotropic part of the stress tensor, which in turn leads to a slight strengthening of the system.

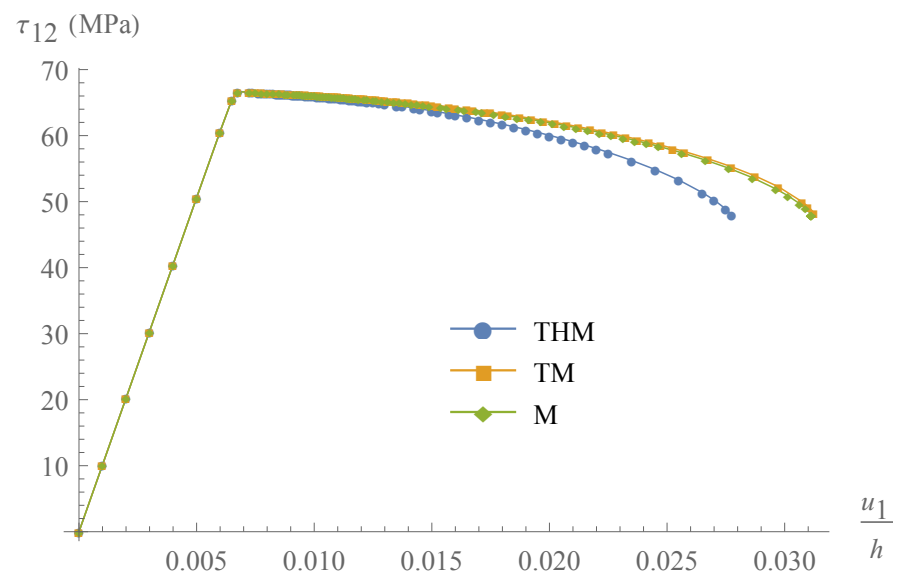

Figure 21: Effect of Thermo-Mechanical (TM) and Thermo-Hydro-Mechanical (THM) couplings on the stress-strain diagram with linear softening law $h_{s}=-0.5(R=10 \mu \mathrm{m})$. 


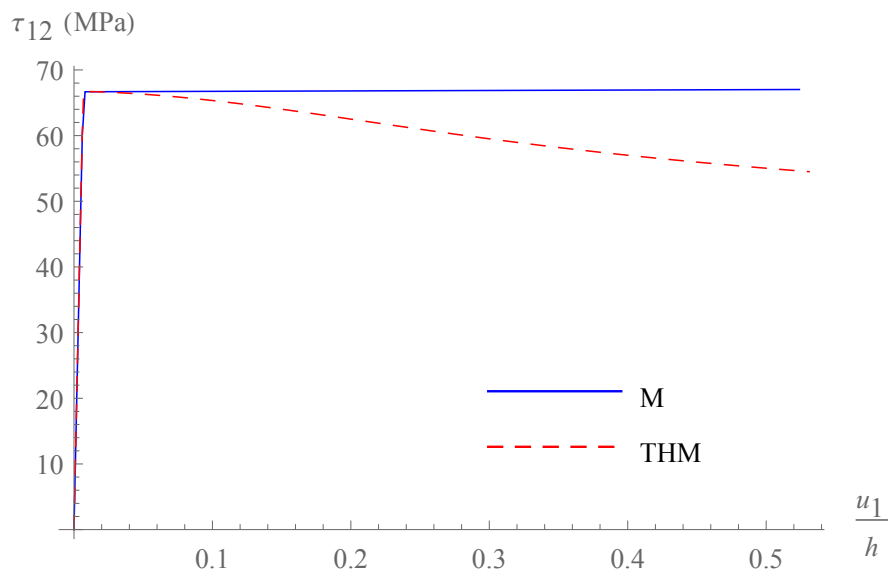

Figure 22: Effect of Thermo-Hydro-Mechanical (THM) couplings on the stress-strain diagram assuming perfect plasticity $(\mathrm{R}=10 \mu \mathrm{m})$.

\subsection{Effect of the thermal pressurization coefficient}

Thermal pressurization is a weakening mechanism that has been largely studied in the context of earthquakes in order to explain the reduction of fault strength with slip (Sibson, 1973, Lachenbruch, 1980, Rempel and Rice, 2006). This phenomenon is due to the discrepancy between thermal expansion coefficients of the solid matrix and pore fluids. Frictional heat induces a pore fluid pressure increase that results in a decrease of the effective mean stress. This weakening mechanism is controlled by the thermal pressurization coefficient $\Lambda$.

In the following, we investigate the influence of the thermal pressurization coefficient, $\Lambda$, on the mechanical behaviour of the system. This coefficient depends on many parameters, such as the nature of the material and of the pore fluid, the stress state and the temperature among others. Previous studies have proposed a wide range of values for $\Lambda$. For instance in (Palciauskas and Domenico, 1982), the authors take a value of $0.59 \mathrm{MPa} /{ }^{\circ} \mathrm{C}$ for Kayenta sandstone, while in (Lachenbruch, 1980), the value taken for an intact rock at great depth is 1.5 $\mathrm{MPa} /{ }^{\circ} \mathrm{C}$. For the analysis, presented in the companion paper, the thermal pressurization coefficient was considered equal to $0.9 \mathrm{MPa} /{ }^{\circ} \mathrm{C}$ for a fault at $7 \mathrm{~km}$ depth. Of course, if $\Lambda=0 \mathrm{MPa} /{ }^{\circ} \mathrm{C}$, the response coincides with that of the 
purely mechanical system, and the higher the thermal pressurization coefficient is, the stronger the weakening becomes.

In Fig. 23, we focus on the evolution of pore pressure and temperature in the middle of the shear band. The temperature evolution shows an exponential development and is slightly influenced by the value of the thermal pressurization coefficient. For $\Lambda=2 \mathrm{MPa} /{ }^{\circ} \mathrm{C}$, the increase of temperature is faster than for lower thermal pressurization coefficients, but the final value attained is lower as the shear band width is decreasing faster and thus the maximum shear strain reached is smaller. The pore pressure evolution is more affected than the temperature by the thermal pressurization, as it is acting as a source term in the fluid mass balance equation. When the pore pressure increases, $\tau_{11}^{\prime}$ and $\tau_{33}^{\prime}$ and $\tau_{22}^{\prime}$ decreases in the same way. It results in a decrease of the mean effective normal stress and causes a weakening of the shear stress.
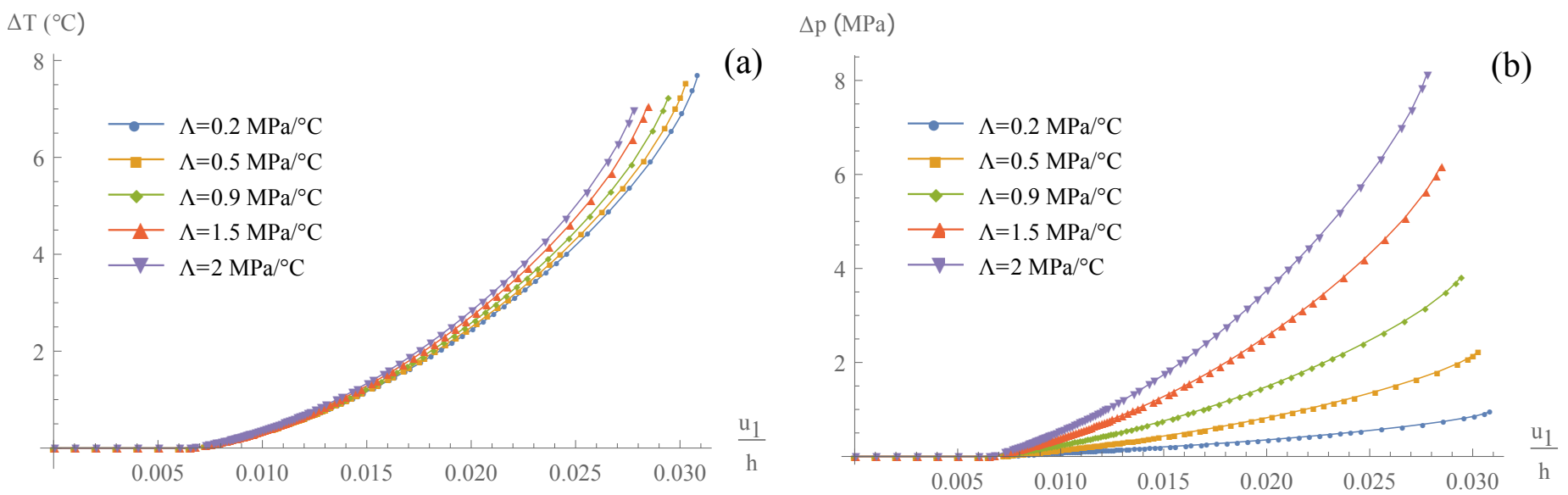

Figure 23: Effect of the thermal pressurization coefficient for $h_{s}=-0.5$ and an internal length $\mathrm{R}=10 \mu \mathrm{m}$ : Evolution of (a) the increase of pore pressure and (b) the increase of temperature, in the middle of the layer.

\subsection{Rate dependency induced by THM couplings}

As shown in Section 2, the mechanical constitutive law that is used in the present paper is rate independent. This means that the constitutive behavior 
of the fault gouge is considered independent of the applied shear velocity at the boundary of the system. However, this does not mean that the overall behavior of the system remains rate-independent when THM couplings are activated due to thermal pressurization and diffusion. It is worth emphasizing that due to the Cosserat formulation, which leads to a finite dissipation and thickness of the localization zone (see Section 3), that the effect of rate dependency due to THM couplings is possible to study.

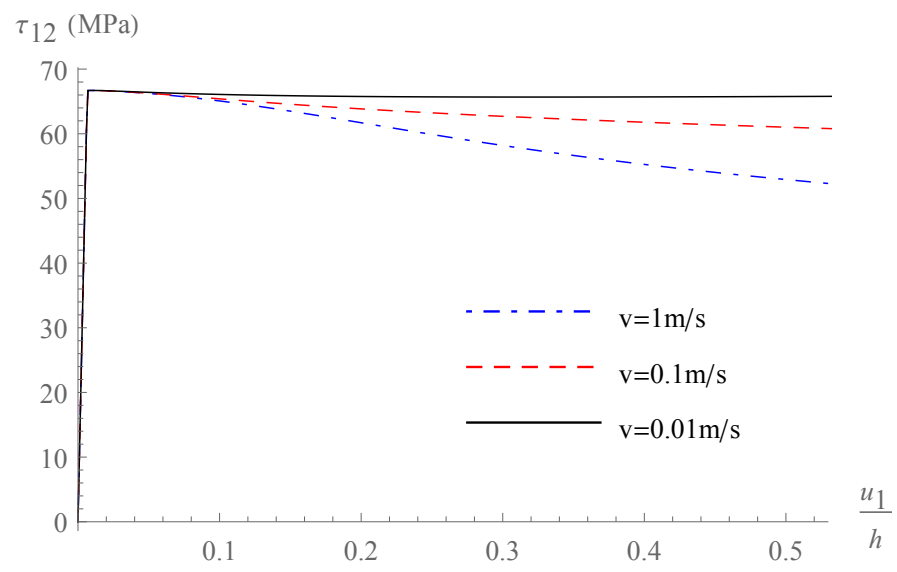

Figure 24: Effect of slip rate on the stress-strain diagram for perfect plasticity $\left(h_{s}=0\right)$, and an internal length $\mathrm{R}=10 \mu \mathrm{m}$

The effect of the imposed slip rate (between $100 \mu \mathrm{m} / \mathrm{s}$ and $1 \mathrm{~m} / \mathrm{s}$ ) is shown in Fig. 24. Constant temperature and pore pressure are imposed at the top and bottom boundaries. The hardening modulus is taken equal to zero (perfect plasticity) in order to illustrate the effect of softening due to thermal pressurization. Notice that for low velocities, no softening is observed as the effect of thermal pressurization is negligible. On the contrary, for higher velocities that reach the co-seismic slip velocities the softening is significant. In Fig. 25, the evolution of the shear band thickness is plotted. For high velocities, a more intense localization is observed. In particular, for slip velocity lower than $0.01 \mathrm{~m} / \mathrm{s}$, no localization occurs. The reason is that due to the low shear velocity, temper470 ature and pore pressure increase rates are low, allowing diffusion to dominate, 


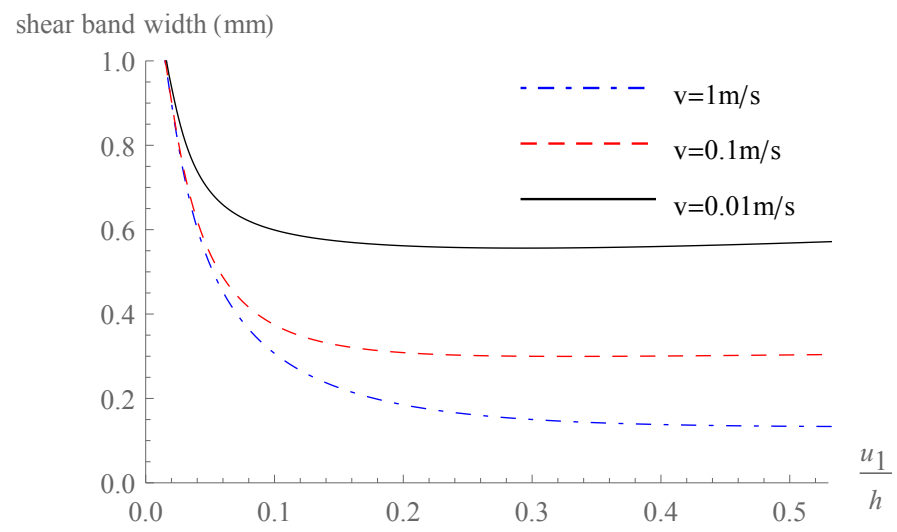

Figure 25: Effect of slip rate on the shear band thickness for perfect plasticity $\left(h_{s}=0\right.$ and $R=10 \mu \mathrm{m})$.

which inhibits localization. In other words the source term is counterbalanced by diffusion, which leads eventually to a steady state (constant pressure and temperature profiles).

During the nucleation of an earthquake, the slip accelerates to reach a maximum velocity of about $1 \mathrm{~m} / \mathrm{s}$. The total slip required to reach that speed is in the order of a few millimeters (Segall and Rice, 2006). Therefore, Fig. 24 shows that THM couplings can greatly affect the fault behavior during this phase (after a sufficient slip necessary for thermal pressurization to become significant). Moreover, the slip along the fault is not homogeneous (Wald and Heaton, 1994) and at the border of the slip patch, the displacements are much smaller than in the middle. The large difference of shear band thickness obtained in Fig. 25 can explain the heterogeneity of localization thickness observed along the same fault as the slip is highly heterogeneous. For example, two drilling projects at different locations in the Chelungpu fault found very different sizes of the local485 ization zone. Boullier et al. (2009) found a principal slip zone of 3-20mm thick, whereas Heermance et al. (2003) observed a PSZ 50-300 $\mu \mathrm{m}$ in the same range as for the exhumed part of the Punchbowl fault. 

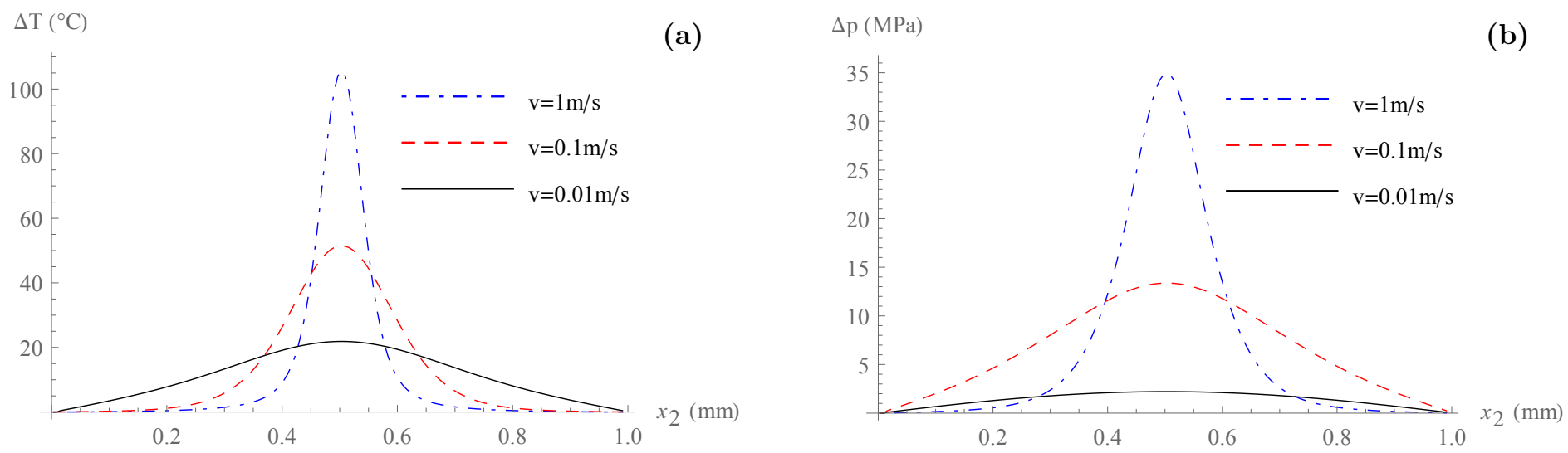

Figure 26: Effect of slip rate for perfect plasticity $\left(h_{s}=0\right)$, and an internal length $R=10 \mu \mathrm{m}$ on (a) the temperature increase profile (b) the pore pressure increase profile, at $\frac{u_{1}}{h}=0.5$

\subsection{Exponential softening law}

The computations presented in Subsection 3.4 with an exponential softening are performed again with the addition of the THM couplings. We present here the results for different values of the internal length. In Fig. 27, the stressdisplacement diagram is plotted. We observe that when THM couplings are considered, the shear stress $\tau_{12}$ does not reach a plateau, but keeps decreasing, as the heat produced by plastic dissipation continues to reduce the strength due to thermal pressurization. Nevertheless, the mechanical and THM models follow the same decrease at the beginning of the softening and then diverge with increasing shear deformation. As expected, thermal pressurization does not play a significant role on the nucleation of the instability, but rather on the evolution of the slip and on energy dissipation (Segall and Rice, 2006).

In Fig. 28, the evolution of the shear band thickness is investigated for the exponential softening. The THM couplings tend to make the band thinner and have more effect on the residual thickness than on the minimum. We notice that even though the shear stress does not reach a steady state, the width of the band does. The minimum and the residual values for various internal lengths are represented in Fig. 29, and we observe also a linear evolution for the two 


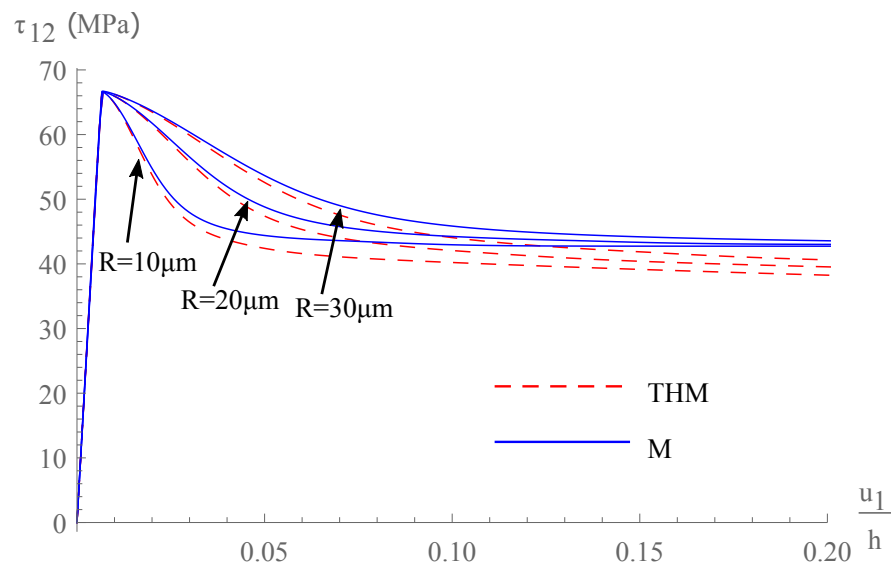

Figure 27: Effect of THM couplings on the stress-strain diagram for an exponential hardening $\left(\mu_{\text {res }}=0.3\right.$ and $\left.\gamma_{e}=0.2\right)$ and different internal lengths $(\mathrm{R}=10,20$ and $30 \mu \mathrm{m})$ considering a slip rate of $1 \mathrm{~m} / \mathrm{s}$.

estimations with THM couplings.

For $R=10 \mu \mathrm{m}$, we compare these minima and residual values of the shear band width with the values obtained for perfect plasticity (Fig. 25). In both cases, the shear rate applied is $1 \mathrm{~m} / \mathrm{s}$. The evolution of the thickness is similar as we first observe a decrease followed by an increase. Still, the values of the minimum and residual thickness are different. In the case of perfect plasticity, the minimum value is $124 \mu \mathrm{m}$ and the residual thickness is $128 \mu \mathrm{m}$ (Fig. 25). They are larger than the values obtained considering an exponential softening: $69 \mu \mathrm{m}$ and $85 \mu \mathrm{m}$ (Fig. 28). Thus, we can conclude that the localization process also depends on the history of the loading.

Finally, thermal pressurization does not greatly affect the shear band thickness when the material softening alone is significant (Fig. 28). However, if the mechanical behavior is perfectly plastic, no localization is observed unless THM couplings are considered and, therefore, the thermal pressurization plays a crucial role in the control of the size of the principal slip zone. In both cases, the internal length required to simulate a shear band thickness with a size in agreement with observations of the Punchbowl fault (see Section 3.4) is one order 


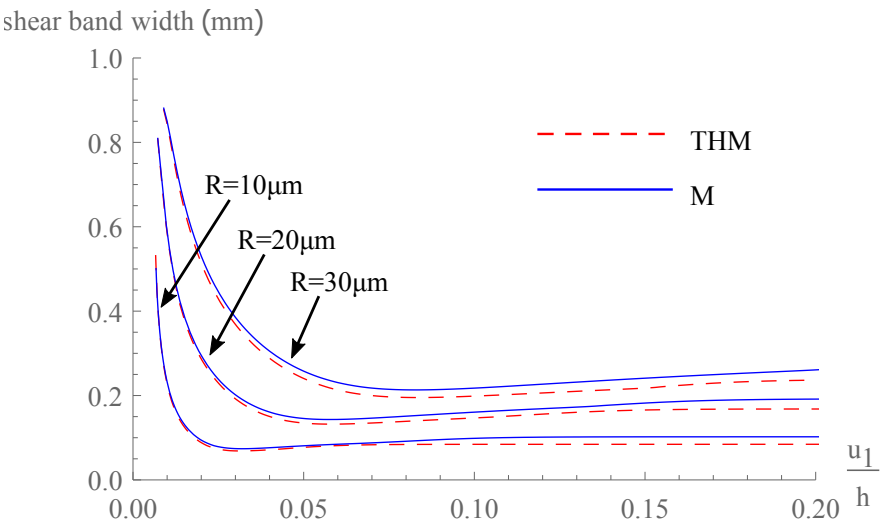

Figure 28: Effect of THM couplings on the shear band thickness evolution for an exponential hardening $\left(\mu_{\text {res }}=0.3\right.$ and $\left.\gamma_{e}=0.2\right)$ and different internal lengths $(\mathrm{R}=10,20$ and $30 \mu \mathrm{m})$.

of magnitude larger than the mean grain size and is closer to $D_{70}$ (mesh size corresponding to $70 \%$ of passing mass in sieve analysis). Note that most of the existing laboratory experiments and DEM simulations have been performed with a relatively narrow grain size distribution. For this case, taking a Cosserat length of the order of $D_{50}$ is valid (see (Mühlhaus and Vardoulakis, 1987)). We expect in the future to bring new experimental results on the effect of wider grain size distribution on the shear band thickness.

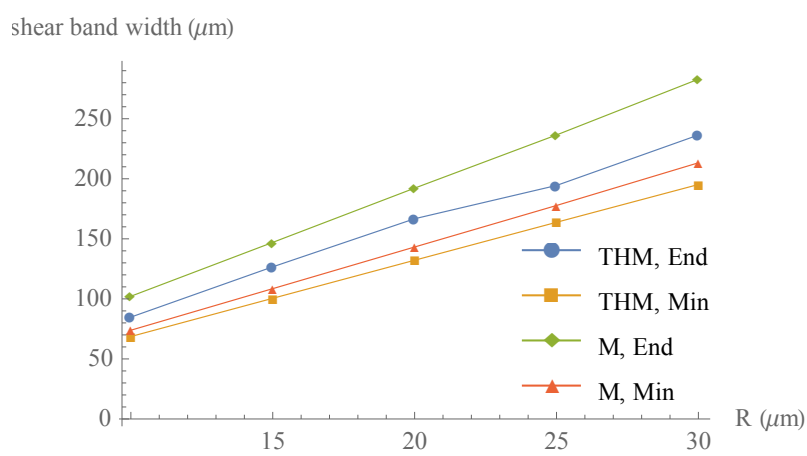

Figure 29: Effect of THM couplings on the evolution of the minimum shear band thickness and the residual one with the internal length. 


\section{Comparisons with results of the linear stability analysis}

Strain localization is seen as an instability of the inelastic macroscopic constitutive laws. The initial homogeneous state becomes unstable (bifurcation), which triggers the onset of localization (Rudnicki and Rice, 1975). Moreover, we can assess the size of the localized zone by analyzing the dominant wavelength of the perturbation modes. In (Rice et al., 2014), the system of equations presents a critical wavelength over which all perturbations are unstable. The band width is estimated as half the value of the critical wavelength. In the companion paper (Rattez et al., 2017a) (see also Sulem et al. (2011), Veveakis et al. (2013)), the use of a Cosserat continuum with inertia terms enables us to have a stability diagram (Lyapunov exponent (Lyapunov, 1992) plotted versus the wavelength of the perturbation) that does not present a vertical asymptote. Thus, the shear band thickness is calculated from the value of the wavelength of the perturbation that grows the fastest (the maximum of the Lyapunov exponent).

In this section, we will compare the values of the shear band thickness ob${ }_{545}$ tained from the LSA and the one obtained from FEM computations. For that, at each time step of the numerical computation, we define an associated system of a homogeneous state for which LSA is performed. This associated homogeneous system is assumed to be in a softening state obtained from the slope of the numerical stress-strain $\left(\tau_{12}\right.$ in function of $\left.u_{1} / h\right)$ curve at the considered time step. In this paper, a quasi-static analysis is conducted and therefore, no maximum in the LSA is observed in this case. Therefore, in the following, the shear band thickness obtained in the LSA is evaluated as in Rice et al. (2014). Moreover, the methodology of comparison, which is explained in detail in Appendix C, permits us to understand how the loss of homogeneity in the system further influences the evolution of the localization process.

\subsection{Comparisons for mechanical simulations with linear softening}

In this section, we compare the evolution of the shear band thickness obtained numerically as described in 3.3 with the one predicted by the linear 
stability analysis for purely mechanical examples and considering a linear evolution of the friction coefficient. The parameters chosen are defined in 3.1 and we study the effect of the internal length and the height of the layer on the comparison.

In Fig. 30, we consider internal lengths ranging from $10 \mu \mathrm{m}$ to $30 \mu \mathrm{m}$ and heights of the layer of 1 or $2 \mathrm{~mm}$. For a height of $1 \mathrm{~mm}$ and $R=10 \mu \mathrm{m}$ (Fig. 30 (a)), the thickness obtained at the initiation of the localization process is identical for the LSA and the finite element simulation as this state of the layer is the closest to a homogeneous state of deformation as considered in the definition of the associated system for which the linear analysis is applied. Nevertheless, for $\mathrm{R}=20$ and $30 \mu \mathrm{m}$ and the same height (Fig. 30 (b) and (c)), we do not observe the same behavior. This apparent discrepancy is due to the height of the layer which is not sufficiently large to have vanishing couple stresses in the profile. The associated problem differs significantly from the original one in that case and leads to different values of the shear band thickness. For a height of $2 \mathrm{~mm}$ (Fig. 30 (e) and (f)), this is no longer an issue and the size of the bands obtained 575 at the nucleation by LSA and FEM match.

After the initiation of localization, the LSA tends to overestimate the thickness of the shear band, as the state of deformation in the original system is no longer homogeneous. Yet, the difference remains small and tends to decrease as the height of the layer increases, but if the height gets too large, we face 580 convergence problems as the softening branch gets too steep. The tendency at the end of the numerical simulations to have a thickness that decreases to the value of the internal length is also captured by the LSA. 

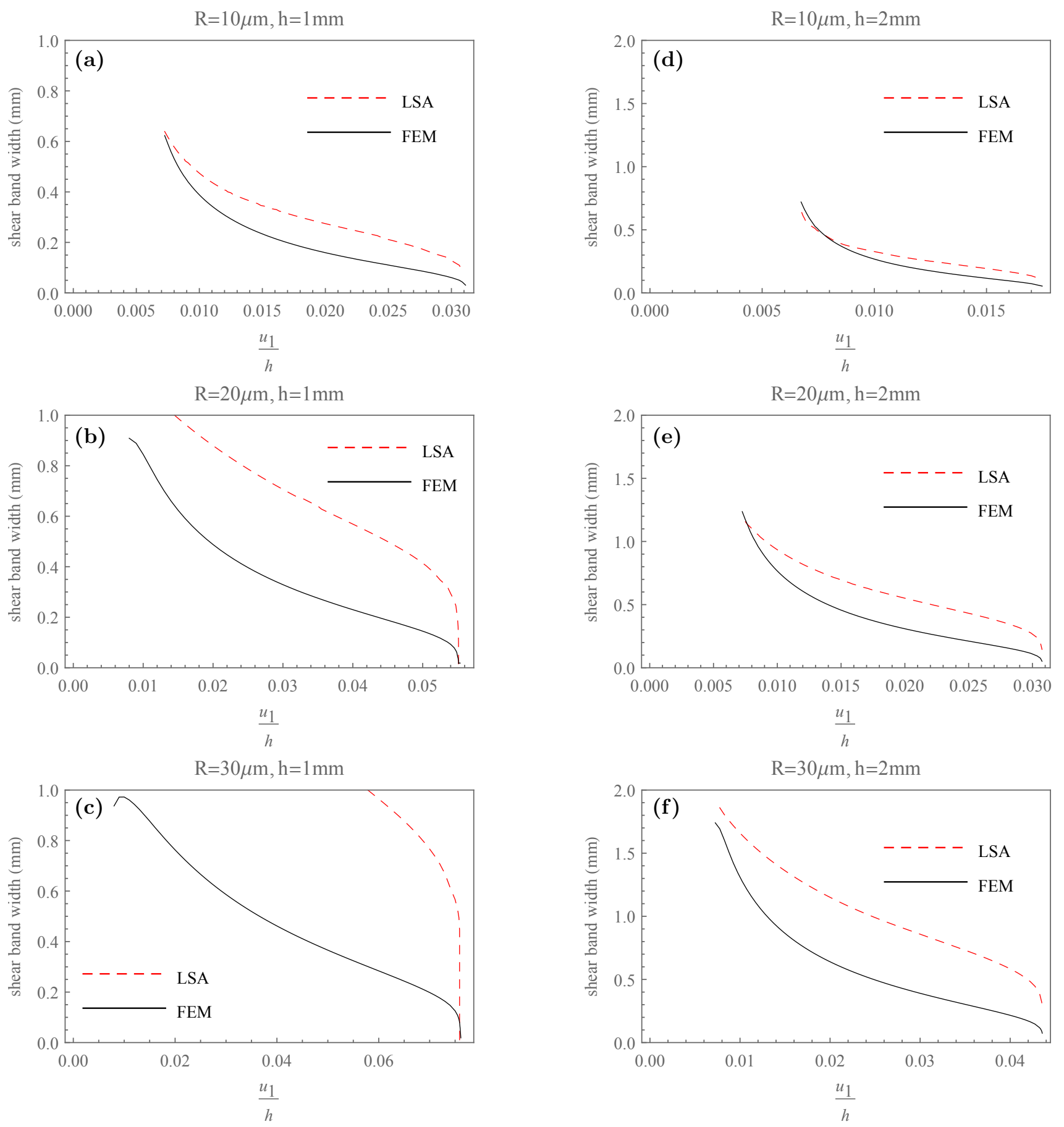

Figure 30: Comparisons of the shear band thickness evolution for internal lengths between 10 and $30 \mu \mathrm{m}$ and two heights of the layer $h, 1$ and $2 \mathrm{~mm}$ using a linear evolution of the friction coefficient. 


\subsection{Comparisons for mechanical simulations with exponential hardening}

The comparison between LSA and FEM is also made for the case of an

585

the simulations are presented in section 3.4. We consider here a residual friction coefficient of 0.3 and a factor in the exponential $\gamma_{e}$ of 0.2 . As in the preceding section, the analysis focuses on the effect of the internal length and of the height of the layer.

the LSA fit well the numerical ones for internal lengths of 10 and $20 \mu \mathrm{m}$ (Fig. 30 (a), (b), (d) and (e)). It is not the case for $R=30 \mu \mathrm{m}$ as the distribution of couple stress $\mu_{32}$ over the layer doesn't reach a value close to zero at the boundary, even for a height of $2 \mathrm{~mm}$ (Fig. 30 (c) and (f)). Note that in Fig. 31(d) $(R=10 \mu \mathrm{m}$ exhibit the formation of two symmetrical shear bands at a quarter and three quarters of the layer thickness. Therefore the comparison with LSA is performed by considering only half of the layer (of thickness $1 \mathrm{~mm}$ ) in order to capture only one band.

600

After the nucleation, the shear band size decrease is well captured by the LSA. But as the thickness approaches its minimum value, the two curves begin to diverge significantly. The level of deformation is higher than the one corresponding to a linear softening of the friction coefficient, which explains this apparent contradiction with the section above. Moreover, when the shear stress tends to the residual value, the hardening modulus tends to zero. Thus, the shear band thickness obtained by the linear analysis grows indefinitely.

\subsection{Comparisons for THM simulations}

The comparison of LSA and FEM results is then performed for the system of the infinite sheared layer considering Thermo-Hydro-Mechanical couplings with the set of parameters described in Table 3. Only one example for a linear evolution of the friction coefficient and one example for the exponential law are presented here. 

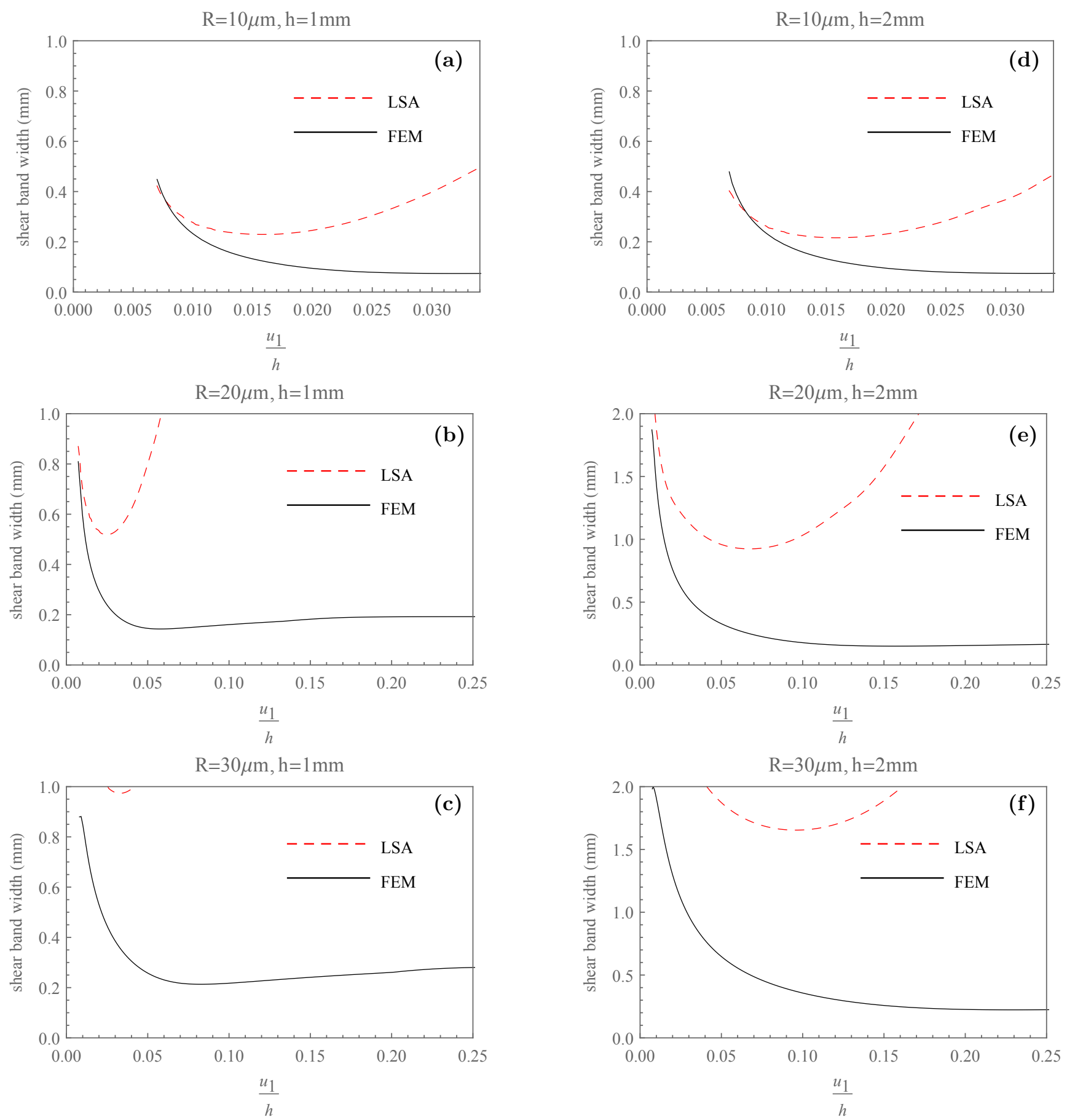

Figure 31: Comparisons of the shear band thickness evolution for internal lengths between 10 and $30 \mu \mathrm{m}$ and two heights of the layer $h, 1$ and $2 \mathrm{~mm}$ using an exponential evolution of the friction coefficient. 
In Fig. 32, the evolution of the shear band size is plotted for an internal length of $10 \mu \mathrm{m}$, a height of $1 \mathrm{~mm}$ and a hardening coefficient $h_{s}=-0.5$ assuming linear softening. The conclusions of section 5.1 remain the same.

In Fig. 33, the evolution of the shear band thickness for an internal length of $10 \mu \mathrm{m}$, a height of $1 \mathrm{~mm}$ for an exponential softening with $\mu_{r e s}=0.3$ and $\gamma_{e}=0.2$ is shown. The initiation and the decrease of the shear band thickness at the beginning of the localization process are well captured by the LSA. When the deformation increases, the two lines diverge as it is observed for the purely mechanical system (Fig. 31). However, unlike the mechanical system, when the shear stress converges to a residual value and the hardening modulus tends to zero, the linear analysis tends to a finite value in the THM system because the bifurcation is obtained for a positive hardening modulus (Rattez et al., 2017a). This value of the final shear band thickness obtained by the LSA is larger than the height of the layer and one order of magnitude larger than the residual thickness obtained numerically.

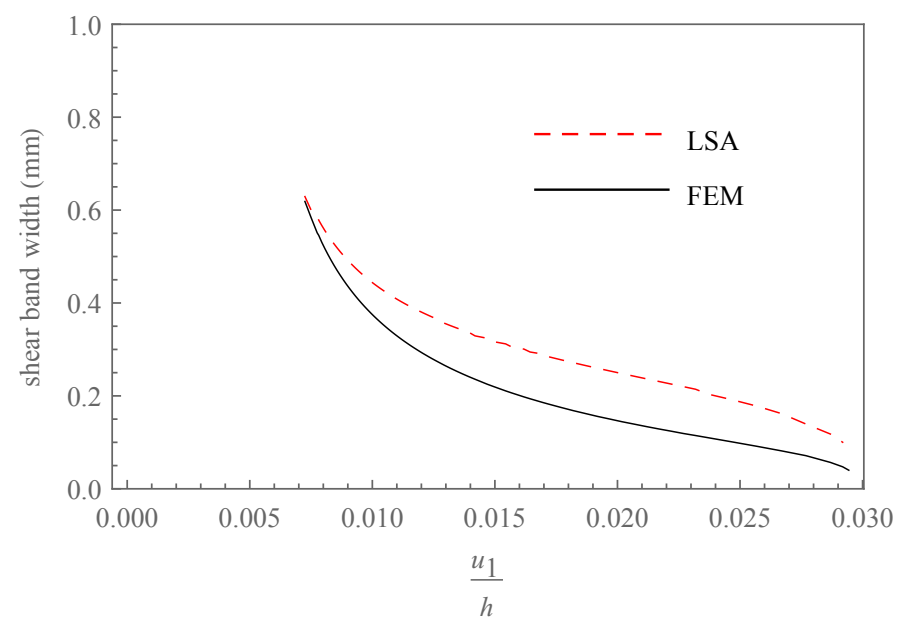

Figure 32: Comparison of LSA and FEM results for the shear band thickness evolution for the THM model assuming an internal length $R=10 \mu \mathrm{m}$, a height of $1 \mathrm{~mm}$, a hardening coefficient $h_{s}=-0.5$ and using a linear evolution of the friction coefficient. 


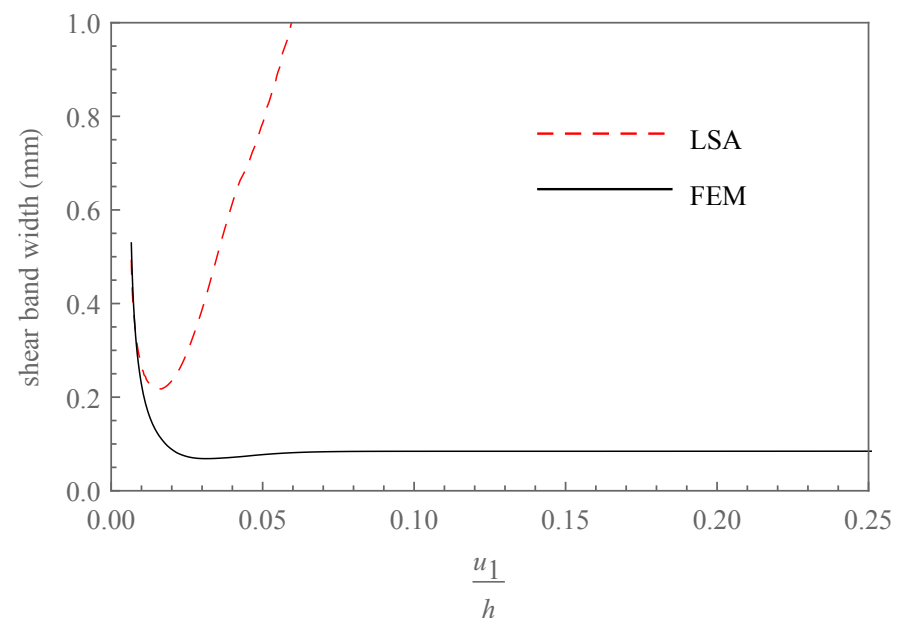

Figure 33: Comparison of LSA and FEM results for the shear band thickness evolution for the THM model assuming an internal length $R=10 \mu \mathrm{m}$, a height of $1 \mathrm{~mm}$ and using an exponential evolution of the friction coefficient $\left(\mu_{r e s}=0.3\right.$ and $\left.\gamma_{e}=0.2\right)$.

\section{Conclusions}

In the present paper, a general three-dimensional Cosserat Finite Element formulation with THM couplings is presented and implemented in a parallel finite element code. This code enables the investigation of the role of the microstructure and THM couplings on the stability and evolution of various geomechanical systems. However, even though the presentation and formulation of the aforementioned model is general, we focus mainly on the behavior of faults. In particular, we study numerically the evolution of the localization zone thickness in a fault gouge and its stress-strain response, which is of major importance regarding earthquake nucleation and energy budget during seismic slip.

Following the mathematical model presented in the companion paper, the various THM couplings that take place during co-seismic slip are considered. As far as the mechanical behavior of the gouge is concerned, an elasto-plastic Cosserat continuum is used. This is a framework of choice when the size of the microstructure plays a fundamental role in the physics of the system (see Part 
I), as it is the case with faults. The dependency of both the shear band thickness and the stress-strain response on various parameters is explored. More specifically, the influence of the size of the microstructure, the hardening evolution, the thermal pressurization coefficient and the slip rate is studied in details.

The comparison of the shear band thicknesses obtained from the linear stability analysis and the finite element simulations has shown that the obtained values are comparable as long as the effects of the induced perturbation of the initial homogeneous state are small enough. It highlights the importance of the induced heterogeneities in the progressive localization process and the relevance to use numerical simulations for quantitative results when studying strain localization. Moreover, the numerical simulations provide additional fundamental information not only on the stress-strain response, but also on the temperature and pore pressure evolution.

Regarding the role of the grain size, the numerical analyses corroborate the analytical findings of Part I. Indeed, numerical analyses show that the evolution of the thickness of the localization zone depends on the size of the grains of the fault core. The larger the grains are the larger the shear band thickness is. Moreover, using an appropriate softening law for fault core materials, it is shown that the final, residual thickness of the localization zone is proportional to the grain size. Considering the example of the Punchbowl fault, $D_{70}$ seems to be more appropriate as internal length for gouge materials presenting a very broad grain size distribution.

Numerical analyses also allow the investigation of the stress-strain response of the system and its dependence on the size of the microstructure. The smaller the grain size is in our model, the steeper the softening branch becomes. This relationship has a direct effect on earthquake nucleation and on the transition from aseismic to seismic slip (Scholz, 2002). This is not a surprising result (cf. the experiments of Marone and Kilgore (1993) on the influence of grain size on the critical slip distance), but this influence is investigated here systematically based on a physical model that can account for the size of the microstructure.

The ability of the Cosserat continuum to provide localization zone of finite 
thickness (contrary to the Cauchy continuum, cf. Section 3.2) makes it possible to examine the influence of the hardening modulus evolution on the shear band thickness. Different softening laws are used, including an exponential softening of the friction coefficient, which is adequate for modeling the mechanical behavior of fault gouges and can be calibrated based on experimental data. Hardening (softening) modulus encapsulates and upscales various micromechanical phenomena and, together with the intrinsic Cosserat lengths, affects the stressstrain response. However, the roles of the hardening modulus and grain size are distinct. It is shown that the hardening modulus controls only the slope of the softening branch, while the grain size controls the localization thickness and thus affects the slope of the softening branch.

Another important factor, whose role was explored in detail in the companion paper, is thermal pressurization. This destabilizing mechanism is practically negligible at the nucleation phase of seismic slip and its effect is rather noticed during rapid co-seismic slip, where temperature increases significantly due to friction. The magnitude of this phenomenon is described through the, so-called, thermal pressurization coefficient, which expresses the increase of pore pressure in the gouge due to temperature increase. The numerical analyses confirm and extend the qualitative findings presented in Part I. Under high shearing deformation rates, it is shown that the thermal pressurization coefficient has a direct impact on the post peak evolution of the stress-strain response. Increased softening is observed for higher values of the aforementioned coefficient leading to more localized deformation. The effect of thermal pressurization is pronounced even when no-hardening/softening is considered, showing the significance of the mechanism in modeling and energy budget during seismic slip.

A substantial result exhibited in this paper is that both the softening, postpeak behavior and the shear band thickness depend on the applied velocity, showing thus rate dependency despite assuming a rate-independent constitutive description of the material. Thermal pressurization is again the central mechanism for this apparent rate-dependent behavior. In particular, the applied velocity controls the local dissipation rate due to friction and consequently the 
pore pressure increase rate. The pore pressure increase rate is controlled in parallel by the thermal and pore-pressure diffusion mechanisms, which introduce a characteristic time scale. Under high velocities the characteristic diffusion rate is small in comparison to the dissipation rate, which has as a result a more localized profile of deformation and a steeper post-peak softening branch. However, under low velocities, the diffusion mechanisms dominate, leading to a quasi homogeneous deformation profile without significant global softening if perfect plasticity is assumed for the fault gouge.

In this study, we focused on the role of the most prominent Thermo-Hydro715 Mechanical couplings related seismic slip (see also Viesca and Garagash (2015)). Yet, the present model can be extended to account for other weakening processes observed in fault zones, such as grain cataclasis and chemical reactions, among others. The former can be modeled by adequately varying the internal length of the Cosserat model (Bauer, 2016) and the latter by adding additional terms to 720 the constitutive description of the Cosserat material and the mass and energy balance equations (Sulem and Famin, 2009, Brantut and Sulem, 2012, Veveakis et al., 2013, Platt et al., 2015). Such extensions will provide in future work finer descriptions of important mechanisms that take place at different time and length scales and give valuable results regarding seismic slip and energy 725 dissipation. 


\section{References}

Alsaleh, M. I., 2004. Numerical Modeling of Strain Localization in Granular Materials Using Cosserat Theory Enhanced With Microfabric Properties. Ph.D. thesis, Louisiana State University.

An, L. J., Sammis, C. G., 1994. Particle size distribution of cataclastic fault materials from Southern California: A 3-D study. Pure and Applied Geophysics PAGEOPH 143 (1-3), 203-227.

Andrews, D. J., 2002. A fault constitutive relation accounting for thermal pressurization of pore fluid. Journal of Geophysical Research: Solid Earth 107 (B12), 2363.

URL http://doi.wiley.com/10.1029/2002JB001942

Bauer, E., 2016. Simulation of the Influence of Grain Damage on the Evolution of Shear Strain Localization. In: Continuous Media with Microstructure 2. pp. 231-244.

Benallal, A., Comi, C., 2003. Perturbation growth and localization in fluidsaturated inelastic porous media under quasi-static loadings. Journal of the Mechanics and Physics of Solids 51 (5), 851-899.

Besuelle, P., Chambon, R., Collin, F., 2006. Switching deformations modes in post-localization solutions with a quasi-brittle material. journal of materials and structures 1 (7), 1115-1134.

Boullier, A. M., Yeh, E. C., Boutareaud, S., Song, S. R., Tsai, C. H., 2009. Microscale anatomy of the 1999 Chi-Chi earthquake fault zone. Geochemistry, Geophysics, Geosystems 10 (3).

Brantut, N., Sulem, J., 2012. Strain Localization and Slip Instability in a Strainics 79 (3), 031004.

URL http://link.aip.org/link/JAMCAV/v79/i3/p031004/s1\{\&\}Agg=doi 
Chambon, G., Schmittbuhl, J., Corfdir, A., 2002. Laboratory gouge friction: Seismic-like slip weakening and secondary rate- and state-effects. Geophysical Research Letters 29 (10), 4.

Chambon, R., Caillerie, D., Viggiani, G., 2004. Loss of uniqueness and bifurcation vs instability: some remarks. Revue Française de Génie Civil 8 (5-6), $517-535$.

Chambon, R., Crochepeyre, S., Charlier, R., 2001. An algorithm and a method to search bifurcation points in non-linear problems. International Journal for Numerical Methods in Engineering 51 (3), 315-332.

Chester, F. M., Chester, J. S., 1998. Ultracataclasite structure and friction processes of the Punchbowl fault, San Andreas system, California. Tectonophysics 295 (1-2), 199-221.

Chester, J. S., Chester, F. M., Kronenberg, A. K., 2005. Fracture surface energy of the Punchbowl fault, San Andreas system. Nature 437 (7055), 133-136.

Collin, F., Chambon, R., Charlier, R., 2006. A finite element method for poro mechanical modelling of geotechnical problems using local second gradient models. International Journal for Numerical Methods in Engineering 65 (11), 1749-1772.

de Borst, R., 1991. Simulation of Strain Localization: a Reappraisal of the Cosserat Continuum. Engineering Computations 8 (4), 317-332.

de Borst, R., Sluys, L., Mühlhaus, H.-B., Pamin, J., feb 1993. Fundamental Issues in Finite Element Analyses of Localization of Deformation. Engineering Computations 10 (2), 99-121.

Di Toro, G., Han, R., Hirose, T., De Paola, N., Nielsen, S., Mizoguchi, K., Ferri, F., Cocco, M., Shimamoto, T., 2011. Fault lubrication during earthquakes. Nature 471 (7339), 494-498.

URL http://www.nature.com/doifinder/10.1038/nature09838 
Ebrahimian, B., Noorzad, A., Alsaleh, M. I., 2012. Modeling shear localization along granular soil-structure interfaces using elasto-plastic Cosserat continuum. International Journal of Solids and Structures 49 (2), 257-278.

Ehlers, W., Scholz, B., 2007. An inverse algorithm for the identification and the sensitivity analysis of the parameters governing micropolar elasto-plastic granular material. Archive of Applied Mechanics 77 (12), 911-931.

Garagash, D. I., 2012. Seismic and aseismic slip pulses driven by thermal pressurization of pore fluid. Journal of Geophysical Research: Solid Earth 117 (4).

Garagash, D. I., Rudnicki, J. W., 2003. Shear heating of a fluid-saturated slipweakening dilatant fault zone: 1. Limiting regimes. Journal of Geophysical Research 108 (B22121), 1-19.

Gaston, D., Newman, C., Hansen, G., Lebrun-Grandié, D., 2009. MOOSE: A parallel computational framework for coupled systems of nonlinear equations. Nuclear Engineering and Design 239 (10), 1768-1778.

Germain, P., 1973. La méthodes des puissances virtuelles en mécanique des milmieux continus - 1. Théorie du second gradient. Journal de Mecanique Theorique et Appliquee 12 (2), 235-274.

Godio, M., Stefanou, I., Sab, K., Sulem, J., 2015. Dynamic finite element formulation for Cosserat elastic plates. International Journal for Numerical Methods in Engineering 101 (13), 992-1018.

Godio, M., Stefanou, I., Sab, K., Sulem, J., nov 2016. Multisurface plasticity for Cosserat materials: Plate element implementation and validation. International Journal for Numerical Methods in Engineering 108 (5), 456-484.

URL http://doi.wiley.com/10.1002/nme.5219

Hall, S. A., Bornert, M., Desrues, J., Lenoir, N., Pannier, Y., Viggiani, G., Bésuelle, P., 2010. Discrete and continuum analysis of localised deformation in 
sand using X-ray $\mu \mathrm{CT}$ and volumetric digital image correlation. Géotechnique $60(5), 315-322$.

Heermance, R., Shipton, Z. K., Evans, J. P., 2003. Fault structure control on fault slip and ground motion during the 1999 rupture of the Chelungpu fault, Taiwan. Bulletin of the Seismological Society of America 93 (3), 1034-1050.

Huang, W., Bauer, E., 2003. Numerical investigations of shear localization in a micro-polar hypoplastic material. International Journal for Numerical and Analytical Methods in Geomechanics 27 (4), 325-352.

Iordache, M.-M., William, K., 1998. Localized failure analysis in elastoplastic Cosserat continua. Computer Methods in Applied Mechanics and Engineering $151(3-4), 559-586$.

URL http://www.sciencedirect.com/science/article/pii/ S0045782597001667

${ }_{820}$ Issen, K. A., Rudnicki, J. W., 2000. Conditions for compaction bands in porous rock. Journal of Geophysical Research: Solid Earth 105 (B9), 21529.

URL http://doi.wiley.com/10.1029/2000JB900185

Kanamori, H., Brodsky, E. E., aug 2004. The physics of earthquakes. Reports on Progress in Physics 67 (8), 1429-1496.

${ }_{825}$ Khoei, A. R., Yadegari, S., Biabanaki, S. O. R., 2010. 3D finite element modeling of shear band localization via the micro-polar Cosserat continuum theory. Computational Materials Science 49 (4), 720-733.

Lachenbruch, A. H., 1980. Frictional Heating, Fluid Pressure, and the Resistance to Fault Motion. Journal of Geophysical Research: Solid Earth 85 (10), 60976112.

Lyapunov, A. M., 1992. The general problem of the stability of motion. International Journal of Control 55 (3), 531-773. 
Marone, C. J., Kilgore, B., 1993. Scaling of the critical slip distance for seismic faulting with shear strain in fault zones. Nature 362 (6421), 618-621.

Mizoguchi, K., Hirose, T., Shimamoto, T., Fukuyama, E., 2007. Reconstruction of seismic faulting by high-velocity friction experiments: An example of the 1995 Kobe earthquake. Geophysical Research Letters 34 (1).

Mühlhaus, H.-B., 1986. Scherfugenanalyse bei granularem Material im Rahmen der Cosserat-Theorie. Ingenieur-Archiv 56, 389-399.

Mühlhaus, H.-B., Vardoulakis, I., jan 1987. The thickness of shear bands in granular materials. Géotechnique 37 (3), 271-283.

Needleman, A., 1988. Material rate dependence and mesh sensitivity in localization problems. Computer Methods in Applied Mechanics and Engineering $67(1), 69-85$.

Noda, H., Dunham, E. M., Rice, J. R., 2009. Earthquake ruptures with thermal weakening and the operation of major faults at low overall stress levels. Journal of Geophysical Research: Solid Earth 114 (7).

Palciauskas, V., Domenico, P. A., 1982. Characterization of Drained and Undrained Response of Thermally Loaded Repository Rocks. Water Resources Research 18 (2), 281-290.

Papanastasiou, P. C., Vardoulakis, I., jun 1992. Numerical treatment of progressive localization in relation to borehole stability. International Journal for Numerical and Analytical Methods in Geomechanics 16 (6), 389-424.

Papanastasiou, P. C., Zervos, A., 2016. Numerical modelling of strain localization. In: ALERT Doctoral School 2016. Modelling of instabilities and bifurcation in Geomechanics. pp. 133-164. 
Pijaudier, C. G., Bazant, Z. P., 1987. Non local Damage Theory. Journal of engineering mechanics 113 (10), 1512-1533.

URL http://dx.doi.org/10.1061/(ASCE)0733-9399(1987)113:10(1512)

Platt, J. D., Brantut, N., Rice, J. R., 2015. Strain localization driven by thermal decomposition during seismic shear. Journal of Geophysical Research: Solid Earth 120 (6), 4405-4433.

Platt, J. D., Rudnicki, J. W., Rice, J. R., may 2014. Stability and localization of rapid shear in fluid-saturated fault gouge: 2. Localized zone width and strength evolution. Journal of Geophysical Research: Solid Earth 119 (5), 4334-4359.

URL http://doi.wiley.com/10.1002/2013JB010711

Poulet, T., Paesold, M., Veveakis, M., mar 2016. Multi-Physics Modelling of Fault Mechanics Using REDBACK: A Parallel Open-Source Simulator for Tightly Coupled Problems. Rock Mechanics and Rock Engineering, 1-17.

Rattez, H., Stefanou, I., Sulem, J., 2017a. Thermo-Hydro-Mechanical couplings and strain localisation in 3D continua with microstructure . Part I: Theory and linear stability analysis. submitted to Journal of Mechanics and Physics of Solids.

Rattez, H., Stefanou, I., Veveakis, M., Poulet, T., Sulem, J., 2017b. Numerical analysis of strain localization in Rocks with THM couplings: Benchmark tests and microstructure. Submitted to Rock Mechanics and Rock Engineering.

Rempe, M., Smith, S., Mitchell, T., Hirose, T., Di Toro, G., 2017. The effect of water on strain localization in calcite fault gouge sheared at seismic slip rates. Journal of Structural Geology.

Rempel, A. W., Rice, J. R., 2006. Thermal pressurization and onset of melting in fault zones. Journal of Geophysical Research: Solid Earth 111 (9). 
Scholz, C. H., 2002. The mechanics of earthquakes and faulting, second edi Edition. Cambridge.

Scuderi, M. M., Collettini, C., 2016. The role of fluid pressure in induced vs. triggered seismicity: Insights from rock deformation experiments on carbon910 ates. Scientific Reports 6 (April), 1-9.

URL http://dx.doi.org/10.1038/srep24852 
Segall, P., Rice, J. R., 2006. Does shear heating of pore fluid contribute to earthquake nucleation? Journal of Geophysical Research: Solid Earth 111 (9), $1-17$.

Sharbati, E., Naghdabadi, R., 2006. Computational aspects of the Cosserat finite element analysis of localization phenomena. Computational Materials Science 38 (2), 303-315.

Sibson, R. H., 1973. Interactions between Temperature and Pore-Fluid Pressure during Earthquake Faulting and a Mechanism for Partial or Total Stress Relief. Nature 243, 66-68.

Sibson, R. H., 2003. Thickness of the seismic slip zone. Bulletin of the Seismological Society of America 93 (3), 1169-1178.

Sleep, N. H., Richardson, E., Marone, C., 2000. Physics of friction and strain rate localization in synthetic fault gouge. Journal of Geophysical Research: Solid Earth 105 (B11), 25875-25890.

Steacy, S. J., Sammis, C. G., 1991. An Automaton for Fractal Patterns of Fragmentation. Nature 353 (6341), 250-252.

Stefanou, I., Sulem, J., Rattez, H., 2017. Cosserat approach to localization in geomaterials. In: Handbook of Nonlocal Continuum Mechanics for Materials and Structures. Springer.

Sulem, J., Famin, V., 2009. Thermal decomposition of carbonates in fault zones: Slip-weakening and temperature-limiting effects. Journal of Geophysical Research 114 (B3), 1-14.

Sulem, J., Stefanou, I., 2016. Multiphysics couplings and strain localization in geomaterials. In: ALERT Doctoral School 2016. Modelling of instabilities and bifurcation in Geomechanics. pp. 188-213.

URL

http://alertgeomaterials .eu/data/school/2016/

2016\{_\}ALERT\{_\}schoolbook.pdf 
Sulem, J., Stefanou, I., Veveakis, M., feb 2011. Stability analysis of undrained adiabatic shearing of a rock layer with Cosserat microstructure. Granular Matter 13 (3), 261-268.

Sulem, J., Vardoulakis, I., 1990. Bifurcation analysis of the triaxial test on rock specimens. A theoretical model for shape and size effect. Acta mechanica $83(3-4), 195-212$.

Tejchman, J., 2008. Shear Localization in Granular Bodies with Micro-Polar Hypoplasticity. Springer Series in Geomechanics and Geoengineering. Springer Berlin Heidelberg, Berlin, Heidelberg.

URL http://link.springer.com/10.1007/978-3-540-70555-0

Tordesillas, A., Pucilowski, S., Lin, Q., Peters, J. F., Behringer, R. P., 2016. Granular vortices: Identification, characterization and conditions for the localization of deformation. Journal of the Mechanics and Physics of Solids 90, $215-241$.

URL http://dx.doi.org/10.1016/j.jmps.2016.02.032

Tse, S. T., Rice, J. R., 1986. Crustal earthquake instability in relation to the depth variation of frictional slip properties. Journal of Geophysical Research 91 (B9), 9452.

URL http://doi.wiley.com/10.1029/JB091iB09p09452

Unterreiner, P., oct 1994. Contribution à l'étude et à la modélisation numérique des sols cloués : application au calcul en déformation des ouvrages de soutènement. Ph.D. thesis, Ecole Nationale des Ponts et Chaussées.

Vardoulakis, I., sep 1985. Stability and bifurcation of undrained, plane rectilinear deformations on water-saturated granular soils. International Journal for Numerical and Analytical Methods in Geomechanics 9 (5), 399-414.

URL http://doi.wiley.com/10.1002/nag.1610090502

965 Vardoulakis, I., mar 1986. Dynamic stability analysis of undrained simple shear on water-saturated granular soils. International Journal for Numerical and 
Analytical Methods in Geomechanics 10 (2), 177-190.

URL http://doi.wiley.com/10.1002/nag.1610100206

Vardoulakis, I., 1996. Deformation of water-saturated sand : I. uniform undrained deformation and shear banding. Géotechnique 46 (3), 441-456.

Vardoulakis, I., Aifantis, E. C., 1991. A gradient flow theory of plasticity for granular materials. Acta Mechanica 87, 197-217.

Vardoulakis, I., Sulem, J., 1995. Bifurcation Analysis in Geomechanics. Blackie, Glascow.

Veveakis, M., Stefanou, I., Sulem, J., may 2013. Failure in shear bands for granular materials: thermo-hydro-chemo-mechanical effects. Géotechnique Letters 3 (April-June), 31-36.

Viesca, R. C., Garagash, D. I., 2015. Ubiquitous weakening of faults due to thermal pressurization. Nature Geoscience 8 (11), 875-879. URL http://www. nature.com/doifinder/10.1038/ngeo2554

Wald, D. J., Heaton, T. H., 1994. Spatial and Temporal Distribution of Slip for the 1992 Landers, California, Earthquake. Bulletin of the Seismological Society of America 84 (3), 668-691.

Wang, K., Sun, W., Salager, S., Na, S., Khaddour, G., 2016. Identifying Material Parameters for a Micro-Polar Plasticity Model Via X-Ray Micro-Computed Tomographic (Ct) Images: Lessons Learned From the Curve-Fitting Exercises. International Journal for Multiscale Computational Engineering 14 (4), 389-413.

URL http://www.dl.begellhouse.com/journals/61fd1b191cf7e96f, 2b8c00292c2d9c29, 7f3216382af87d2a.html

\section{Appendix A. Determination of the shear band thickness}

In this paper, the plastic strain rate distribution in the layer, $\dot{\gamma}^{p}\left(x_{2}\right)$, is chosen to evaluate the shear band thickness, like previous numerical as it enables 
to follow the instantaneous evolution of the system (Platt et al., 2014, Hall expression the thickness is a Cosine function. However, it is not the only choice and previous authors have used a Gaussian shape approximation of the strain rate profile (Andrews, 2002, Noda et al., 2009, Garagash, 2012, Platt et al., 2014, Rempel and Rice, 2006) at each time step:

$$
\dot{\gamma}^{p}\left(x_{2}\right) \approx \frac{A}{\sqrt{2 \pi} D} e^{-\frac{1}{2}\left(\frac{x_{2}-Y}{D}\right)^{2}}
$$

where $A$ is the amplitude of the maximum plastic strain rate, $D$ is the standard deviation of the Gaussian distribution and $Y$ is the position of the center. From the fitting of $\dot{\gamma}^{p}\left(x_{2}\right)$ with this Gaussian shape function, we define the shear band thickness as twice the full width at half maximum $2 \sqrt{2 \ln (2)} D$.

The trigonometric interpolating function used in the paper is defined by:

$$
\dot{\gamma}^{p}\left(x_{2}\right) \approx B \cdot \chi_{\left[Y-\frac{\lambda}{2} ; Y+\frac{\lambda}{2}\right]}\left(x_{2}\right) \cdot\left[\cos \left(2 \pi \frac{\left(x_{2}-Y\right)}{\lambda}\right)+1\right]
$$

where $B$ is twice the maximum plastic strain rate, $Y$ is the position of the center,

$\lambda$ is the wavelength of the cosine function and $\chi_{\left[Y-\frac{\lambda}{2} ; Y+\frac{\lambda}{2}\right]}\left(x_{2}\right)$ is a rectangular function defined by:

$$
\chi_{\left[Y-\frac{\lambda}{2} ; Y+\frac{\lambda}{2}\right]}\left(x_{2}\right)= \begin{cases}1 & \text { if } x_{2} \in\left[Y-\frac{\lambda}{2} ; Y+\frac{\lambda}{2}\right] \\ 0 & \text { otherwise }\end{cases}
$$

As stated in the paper, the wavelength of the cosine function is interpreted directly as the shear band thickness and allows a clearer link with results of the LSA (Rattez et al., 2017a).

In Fig. A.34, we notice that the interpolation by the two different functions are very similar. To evaluate the goodness of the fit, we determine the adjusted coefficient of determination at each time step in the plastic regime. The mean value over the full localization process of this coefficient for the Gaussian fit is 0.983, whereas the mean value for the cosine fit is 0.995 .

1015

In Fig. A.35, the shear band width evaluated by both approximations is shown for the example of Section 3.1, with a mesh of 80 elements. Furthermore, 


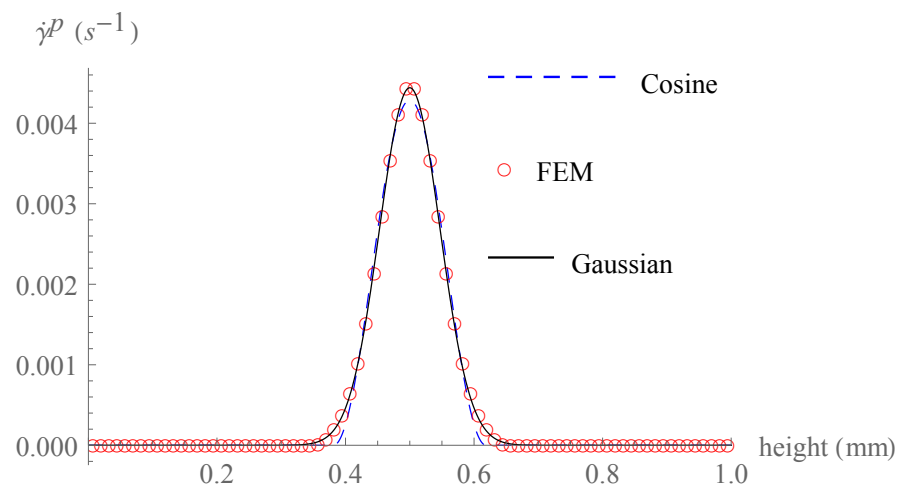

Figure A.34: Interpolation of $\dot{\gamma}^{p}\left(x_{2}\right)$ with a Gaussian and a cosine functions for $\tau_{12}=48 \mathrm{MPa}$ in the example presented in Section 3.1

we have plotted the size of the plastic zone (the zone defined by $F=0$, see Eq. 3.1). All curves exhibit the same tendency: a progressive decrease of the size of the band with accumulated slip. In the paper, the shear band thickness is computed using the Cosine function, as the adjusted coefficient of determination is better.

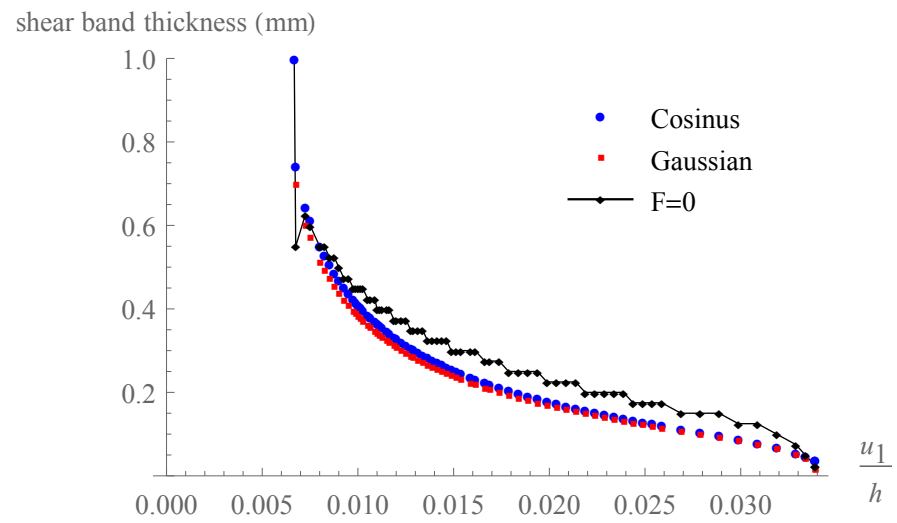

Figure A.35: Interpolation of $\dot{\gamma}^{p}\left(x_{2}\right)$ with a Gaussian and a Cosine function for $\tau_{12}=48 \mathrm{MPa}$ in the example presented in Section 3.1 


\section{Appendix B. Influence of the boundary conditions}

The above numerical results are obtained by imposing zero rotations at the upper and lower boundaries of the layer. This type of boundary condition leads

1025 edges. Consequently, no imperfection in the plastic regime is needed to trigger the localization (de Borst, 1991, Ebrahimian et al., 2012). Moreover, it makes the comparison between the different computations easier as the shear band always develops in the middle, where the rotations can freely develop (Fig. 3).

In Fig. B.36, we present the results of computations imposing a zero moment boundary condition $\left(\mu_{32}=0\right)$ at the top and bottom of the layer. These results are compared with the ones obtained when zero Cosserat rotation is imposed at the boundary $\omega_{3}^{c}=0$. The influence of the position of an imperfection is explored by inserting notches in the geometry. We observe that without any imperfection, numerical errors are sufficient to trigger the localization, which occurs a bit later (in terms of deformation) as compared to the case with zero rotation imposed at the boundary. The position of the shear band is determined by numerical approximations and in general is not located in the middle of the layer (Fig. B.37 (a)). The form of the softening branch in the post localization regime looks the same as only one band develops. To overcome this issue, a notch of $10 \mu \mathrm{m}$ in the middle and at one quarter of the layer is introduced to trigger the localization from the beginning of the softening branch and at the location of the notch (de Borst, 1991).

In the example with a notch at one quarter of the layer and zero moment boundary conditions, the shear band develops at the upper boundary (Fig. B.37 (c)). The notch provokes the initiation of the shear band, but the band migrates then to the upper boundary. Thus, only half a band dissipates the energy of the system. This explains why the softening branch gets much steeper.

Nevertheless if we put a notch in the middle, a band appears at the location of the notch and remains there, but we also observe the emergence of two half bands at the top and bottom boundaries (Fig. B.37 (b)). As two full bands 


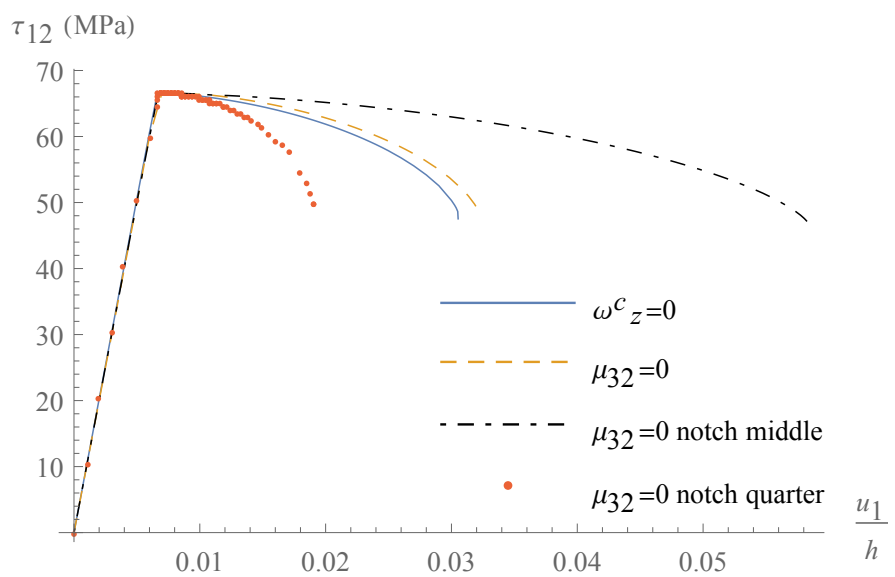

Figure B.36: Effect of the Boundary conditions on the stress-strain diagram

in total dissipate the plastic energy in this case, the softening branch is less steep. Appearence of multiple shear bands is acceptable as the solution of the underlying non-linear mathematical problem is not unique, as explained in Chambon et al. (2001). The authors in this paper have developed a random initialization of the directional searching algorithm to observe different possible solution of a problem in post-localization. As shown in Besuelle et al. (2006) for the case of biaxial loading, different patterns of localized solutions for the same problem are obtained after a random initialization, showing solutions with 1, 2 or 3 bands. 


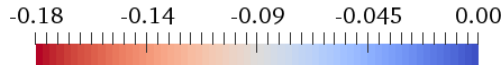

(a)

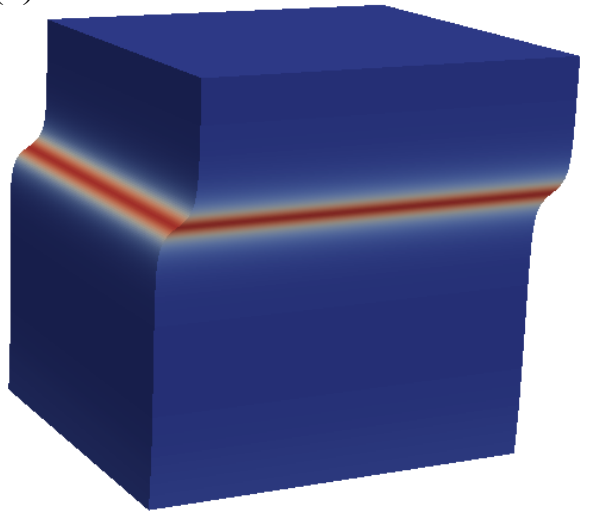

(b)

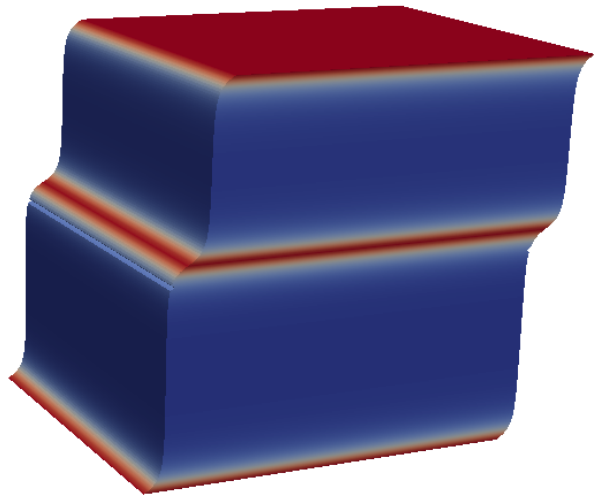

(c)

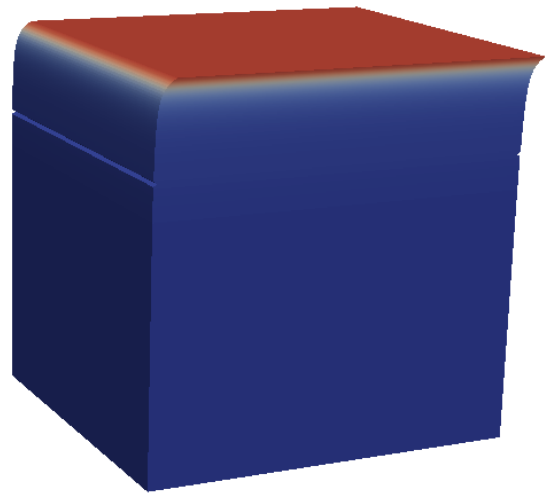

Figure B.37: Cosserat rotations, $\omega_{3}^{c}$, plotted on the deformed mesh for $\tau_{12}=48 \mathrm{MPa}$ of each simulation with $\mu_{32}=0$ imposed at the top and bottom of the layer and for: (a) no notch (b) a notch in the middle of the layer (c) a notch at the quarter of the layer.

\section{Appendix C. Methodology to compare LSA and FEM shear band thickness}

The methodology to assess the shear band thickness performing a Linear Stability Analysis (LSA) is widely developed in the companion paper (Rattez et al., 2017a). In this section, we briefly describe the main results and explain how it is applied in order to compare with the numerical results described in Section 3.3 .

The bifurcation parameter used for the Lyapunov stability analysis in the companion paper is the hardening modulus. For a value of this modulus, the study of the stability diagram enables to obtain an evaluation of the shear band thickness. The way this modulus develops during shearing is calculated from a stress-strain diagram obtained either from an experiment or a numerical simulation. Thus, the first step for the comparison consists in assessing the hardening modulus evolution for each numerical simulations. 
In order to determine the hardening modulus evolution to insert in the LSA, some preliminary calculations are performed for compatibility of the homogeneous associated system for which the LSA is applied. The LSA in the companion paper is performed considering boundary conditions for the perturbation to the layer defined as $\mu_{32}=0$. In other words, the linear stability analysis is valid for a geometry that has no Cosserat effects at its boundary. To fulfill this condition, the evolution of profiles for the shear stresses $\tau_{12}, \tau_{21}$ and the couple stresses $\mu_{32}$ are plotted (see Fig. C.38). From these profiles, we can extract of value of the effective height $h^{*}$ used to calculate the deformation. $h^{*}$ is defined as the minimum distance between the points for which $\mu_{32}$ is less than $0.1 \%$ of the maximum absolute value.

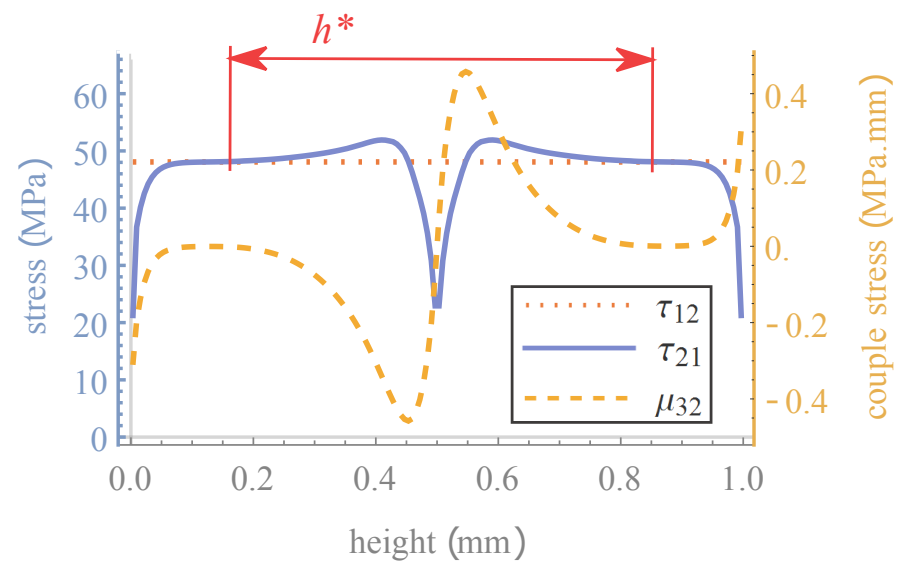

Figure C.38: Profile of shear stresses $\tau_{12}, \tau_{21}$ and couple stress $\mu_{32}$ for $h_{s}=-0.5, R=10 \mu \mathrm{m}$ at the last time step to illustrate the effective height for the calculation of the plastic strain.

The global deformation for the associated system is then calculated using the formula $\gamma_{12}^{*}=\frac{u_{1}\left(\frac{h-h^{*}}{2}\right)-u_{1}\left(\frac{h+h^{*}}{2}\right)}{h^{*}}$. The elastic part of the deformation is retrieved by $\gamma_{12}^{* e}=\frac{\tau_{12}}{G}$. The plastic part of the shear deformation is calculated by $\gamma_{12}^{* p}=\gamma_{12}^{*}-\gamma_{12}^{* e}$ and is equal to the generalized plastic shear strain $\gamma^{* p}$ with the assumptions of small perturbations from the homogeneous state (Cauchy continuum). Furthermore, outside of the shear band, where Cosserat effects are negligible $\left(\tau_{12} \approx \tau_{21}\right.$ and $\left.\mu_{32} \approx 0\right)$, the generalized shear stress $\tau$ is equal to the 
shear stress $\tau_{12}$. We can therefore plot the $\tau-\gamma^{p}$ diagram and from the slope of this diagram obtain an effective hardening modulus that can be used for the LSA (see Fig. C.39).

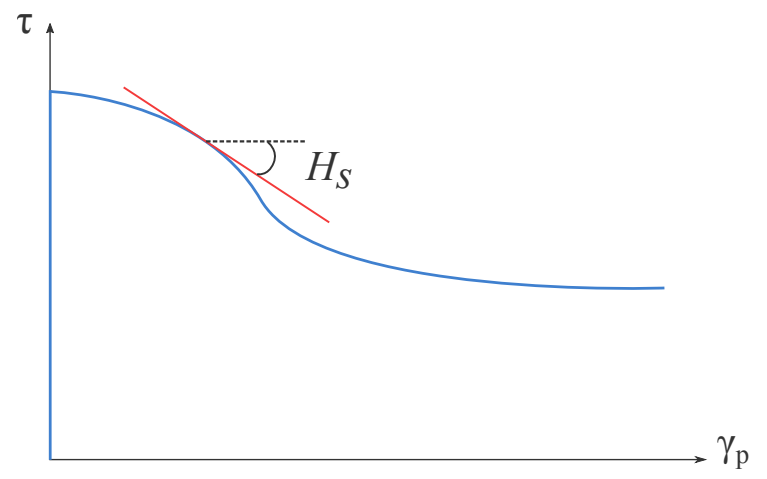

Figure C.39: Schematic diagram of the shear stress invariant versus plastic shear strain invariant showing the geometric interpretation of the hardening modulus $H_{s}$

In order to evaluate the shear band thickness in the companion paper from the linear stability analysis, THM couplings and inertia terms are considered. But these assumptions are not fulfilled for all numerical simulations. When only THM couplings are taken into account(but not the inertia), the stability diagram presents a asymptote instead of a maximum and the shear band thickness is the wavelength associated with the position of this asymptote (see Fig. C.40). If inertia terms are considered for the purely mechanical system, there is a maximum in the stability diagram. Without inertia, it is not possible to perform a LSA for this system as no temporal terms remain in the equations. Therefore, the diagram is plotted with inertia terms, and the values of the minimum unstable wavelength $\lambda_{\min }$ and the wavelength of fastest propagation $\lambda_{\max }$ are extracted (see Figs. C.41 and C.42). 


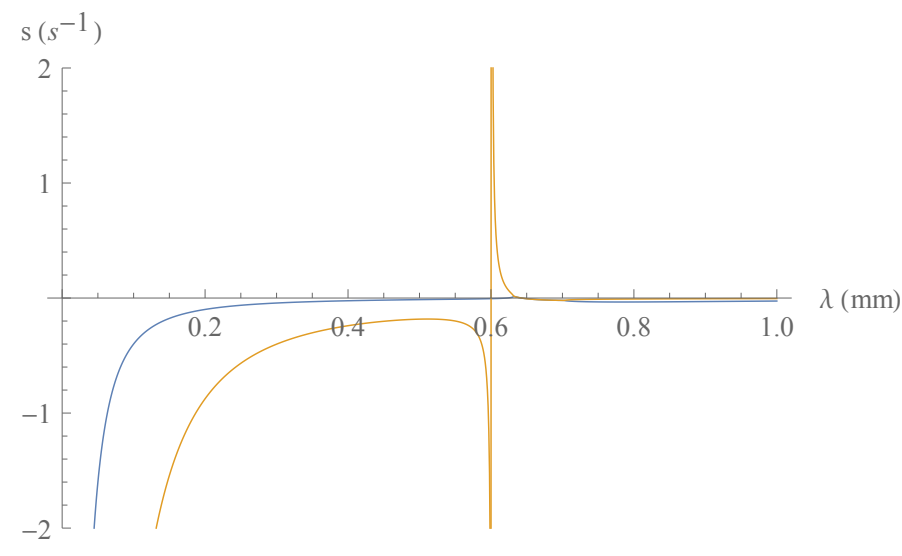

Figure C.40: Example of stability diagram for a THM example neglecting inertia terms showing an asymptote for $\lambda=0.6 \mathrm{~mm}\left(H_{s}=-25 \mathrm{MPa}\right)$.

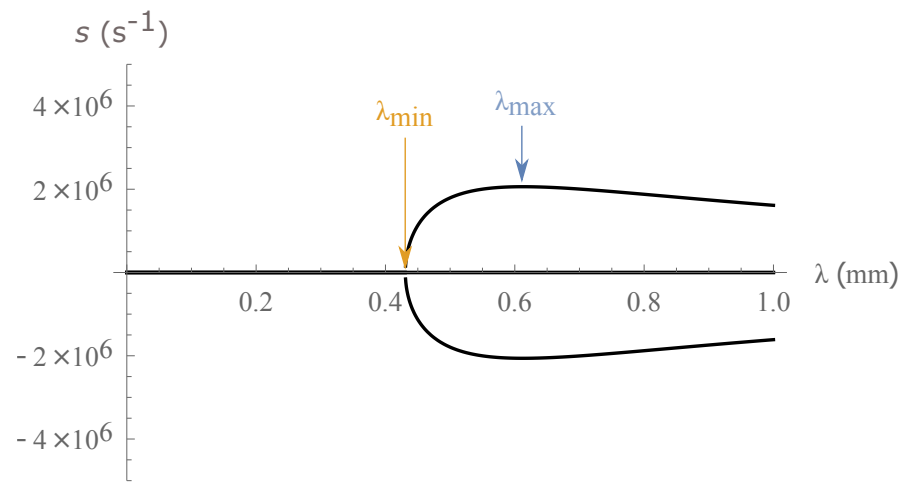

Figure C.41: Example of stability diagram for an isothermal, drained example considering inertia terms showing the wavelength selection $\left(H_{s}=-200 \mathrm{MPa}\right)$. 


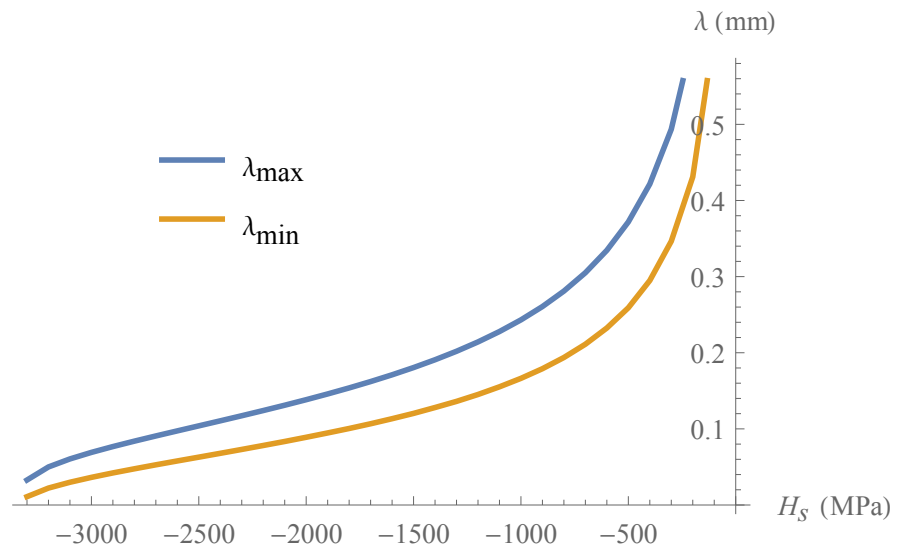

Figure C.42: Example of wavelengths selection evolution with hardening for an isothermal, drained example considering inertia terms. 